\title{
The Dawn Gravity Investigation at Vesta and Ceres
}

\author{
A.S. Konopliv • S.W. Asmar • B.G. Bills • \\ N. Mastrodemos · R.S. Park • C.A. Raymond • \\ D.E. Smith • M.T. Zuber
}

Received: 16 November 2010 / Accepted: 2 June 2011 / Published online: 24 June 2011

(C) Springer Science+Business Media B.V. 2011

\begin{abstract}
The objective of the Dawn gravity investigation is to use high precision X-band Doppler tracking and landmark tracking from optical images to measure the gravity fields of Vesta and Ceres to a half-wavelength surface resolution better than $90-\mathrm{km}$ and $300-\mathrm{km}$, respectively. Depending on the Doppler tracking assumptions, the gravity field will be determined to somewhere between harmonic degrees 15 and 25 for Vesta and about degree 10 for Ceres. The gravity fields together with shape models determined from Dawn's framing camera constrain models of the interior from the core to the crust. The gravity field is determined jointly with the spin pole location. The second degree harmonics together with assumptions on obliquity or hydrostatic equilibrium may determine the moments of inertia.
\end{abstract}

Keywords Dawn · Vesta $\cdot$ Ceres $\cdot$ Gravity $\cdot$ Geophysics

\section{Introduction and Background}

The Dawn mission provides the first opportunity to investigate the interior structures of two dwarf planet sized asteroidal bodies, namely 4 Vesta $(265-\mathrm{km}$ mean radius) and 1 Ceres (476-km mean radius). These asteroids are large, "endmember" examples of planetary embryos, with Vesta once significantly melted and volatile poor, and Ceres less processed in an igneous sense and with a significant volatile content. The interiors of these bodies contain treasure troves of information regarding the range of processes that contributed to accretion

A.S. Konopliv $(\varangle) \cdot$ S.W. Asmar · B.G. Bills · N. Mastrodemos · R.S. Park · C.A. Raymond Jet Propulsion Laboratory, California Institute of Technology, Pasadena, CA 91109, USA

e-mail: alex.konopliv@jpl.nasa.gov

D.E. Smith

Solar System Exploration Division, NASA Goddard Space Flight Center, Greenbelt, MD 20771, USA

M.T. Zuber

Department of Earth, Atmospheric and Planetary Sciences, Massachusetts Institute of Technology,

Cambridge, MA 02139-4307, USA 
of solar system objects from terrestrial planets to ice-rich outer satellites. Previous gravity investigations of other solar system objects have shown varied internal structure of the larger planets (e.g., Zuber et al. 1994, 2000), and the near uniform density distribution of the smaller asteroid 433 Eros (Konopliv et al. 2002). The primary objective of the Dawn gravity experiment is to measure the gravity fields of Vesta and Ceres to $90-\mathrm{km}$ and $300-\mathrm{km}$ halfwavelength resolutions, respectively. Gravity science will provide the primary information on the internal structures of these preserved protoplanets.

Gravity science will be extremely challenging in the Dawn mission because of Dawn's solar electric spacecraft. The frequent thrusting of the solar electric engines represents a non-conservative force that needs to be understood (and in the current mission plan, avoided for extended times) to estimate gravity. Other non-conservative forces such as solar radiation pressure are also increased due to the larger solar arrays required to power a solar electric mission. Gravity is essential to Dawn's science objectives of characterizing the structure and evolution of Vesta (Zuber et al. 2011) and Ceres (McCord et al. 2011). Measurements of gravitational perturbations will provide constraints on the radial distribution of internal mass, including core size and density. Measurement of the orientation of the rotational pole and spin rate may constrain the polar moment of inertia from the measured obliquity (Bills and Nimmo 2011). Although the accuracy and amount of planned tracking data will enable detection of a wobble of the rotation axis, as might be excited by an impact, it is not expected that a detectable wobble will be present, due to the short post-collision damping time (Peale 1976).

Gravity, in combination with improved knowledge of the shapes of Vesta and Ceres (Raymond et al. 2011b) from observations obtained from Dawn's framing camera (Sierks et al. 2011), will provide insight into the distribution of crustal thickness, mechanical properties of the lithosphere, the possible presence of mascons and the nature of impact processes in general.

Dawn's gravity and topography will permit collectively determination of the bulk densities of Vesta and Ceres to better than $1 \%$. The volume of Vesta was found from Hubble images with an uncertainty of about 12\% (Thomas et al. 1997). The most recent mass estimates (see Table 1) have an uncertainty near 2\%. The mass estimates for Vesta are derived from close flybys of other asteroids with Vesta (Michalak 2000; Baer et al. 2008; see home.earthlink.net/ jimbaer1/astmass.txt; Kuzmanoski et al. 2010), the effect of Vesta on the Mars ephemeris (Standish 2001; Pitjeva 2005; Konopliv et al. 2006, 2011; Fienga et al. 2009), the effect of Vesta on the orbit of Eros using the NEAR range data (Konopliv et al. 2002, 2011), and a proposed range of values (Pitjeva and Standish 2009). The Dawn gravity and mission design studies have adopted a value of $G M=17.5 \mathrm{~km}^{3} / \mathrm{s}^{2}$ for Vesta, picked to be in the middle of the probable range of values $\left(17.3-17.8 \mathrm{~km}^{3} / \mathrm{s}^{2}\right)$ from the latest studies. The recent planetary ephemeris and NEAR estimates of the Vesta mass have been improved due to the 10 years of 1-m ranging accuracy to Mars. The NEAR Vesta mass estimate (Konopliv et al. 2011) is an improvement over earlier estimates because systematic errors in the Mars perturbation of the Eros orbit are greatly reduced by the several orders of magnitude improvement in the Mars ephemeris. The Vesta mass estimates from the Dawn mission will be at least $100 \times$ more accurate than the current $2 \%$ uncertainty.

The shape of Ceres was determined to be an oblate spheroid from Hubble images (mean radius $476.2 \pm 1.7 \mathrm{~km}$, Thomas et al. 2005) and from Keck images (mean radius $467.6 \pm$ $2.2 \mathrm{~km}$; Carry et al. 2008). Although individual volume estimates claim uncertainties near $1 \%$, the reported values differ by $5 \%$. As with Vesta, the mass of Ceres is also known from asteroid close encounters (Michalak 2000; Baer et al. 2008) and from the effect on the Mars ephemeris (Standish 2001; Pitjeva 2005; Konopliv et al. 2006, 2011; Fienga et al. 2009) with 
Table 1 Selected mass estimates for Vesta and Ceres

\begin{tabular}{llll}
\hline Asteroid & $\mathrm{GM}\left(\mathrm{km}^{3} / \mathrm{s}^{2}\right)$ & Mass $\left(10^{-12} \mathrm{M}_{\mathrm{A}}\right)$ & Reference \\
\hline Vesta & $18.1 \pm 0.7$ & $136 \pm 5$ & Michalak (2000) \\
& $17.8 \pm 0.2$ & $134.1 \pm 1.5$ & Standish (2001) \\
& $17.84 \pm 0.27$ & $134.4 \pm 2.0$ & Pitjeva (2005) \\
& $18.03 \pm 0.21$ & $135.8 \pm 1.6$ & Konopliv et al. (2006) \\
& $17.51 \pm 0.04$ & $131.9 \pm 0.3$ & Baer et al. (2008) \\
& $17.9 \pm 0.4$ & $135 \pm 3$ & Pitjeva and Standish (2009) \\
& $17.26 \pm 0.04$ & $130.0 \pm 0.1$ & Kuzmanoski et al. (2010) \\
& $17.38 \pm 0.27$ & $131.0 \pm 2.1$ & Konopliv et al. (2011), Mars ephemeris \\
& $17.66 \pm 0.2$ & $133.1 \pm 1.5$ & Konopliv et al. (2011), NEAR \\
& $62.4 \pm 0.2$ & $470 \pm 4$ & Michalak (2000) \\
Ceres & $63.2 \pm 0.2$ & $476.2 \pm 1.5$ & Standish (2001) \\
& $63.08 \pm 0.27$ & $475.3 \pm 2.0$ & Pitjeva (2005) \\
& $62.36 \pm 0.37$ & $469.9 \pm 2.8$ & Konopliv et al. (2006) \\
$63.11 \pm 0.11$ & $475.5 \pm 0.8$ & Baer et al. (2008) \\
& $62.6 \pm 0.4$ & $472 \pm 3$ & Pitjeva and Standish (2009) \\
$62.10 \pm 0.43$ & $467.9 \pm 3.3$ & Konopliv et al. (2011) \\
\hline
\end{tabular}

an accuracy of about 2\% (see Table 1). The Dawn mission will improve knowledge of the mass of Ceres by more than two orders of magnitude in comparison to current estimates.

The Dawn spacecraft will arrive at Vesta in July 2011 and depart July 2012 (Rayman et al. 2011a). During its stay at Vesta, gravity science data will be collected for a total of 50 days in two high altitude mapping orbit (HAMO) phases with spacecraft altitudes near $700-\mathrm{km}$ and for 70 days in one low altitude mapping orbit (LAMO) science phase of the mission with spacecraft altitudes near 200-km. The Dawn spacecraft subsequently arrives at Ceres February 2015 and later collects 21 days of gravity data in the higher altitude orbit and one month of data during the lower altitude orbit. The mission concludes at the end of July 2015. The gravity data consists of Deep Space Network (DSN) $\mathrm{X}$-band Doppler tracking and optical landmark data. A similar approach of combining Doppler tracking and optical landmark data was used for the NEAR mission at Eros. The approach will be similar here, except that, instead of using crater centers, the landmarks are more generalized control points centered at local topography maps, called maplets that cover in a near-uniform manner the visible surface. The topography maps and associated landmark network will be constructed using stereo-photoclinometry (SPC) (Gaskell 2005; Gaskell et al. 2008) and stereo Digital Terrain Maps (DTMs) (Gwinner et al. 2010).

\section{Radiometric and Optical Measurements}

Critically relevant to the Dawn gravity investigation is the telecommunications link between the spacecraft and the Deep Space Network (DSN). The right-hand circularly polarized Xband link is essentially the mission's gravity instrument. The uplink signal, for sending commands to the spacecraft, is centered at $7179 \mathrm{MHz}$ and the downlink signal, for science and engineering telemetry packets is centered at $8435 \mathrm{MHz}$. Dawn's navigators and Radio Scientists utilize this link in its nominal two-way coherent mode for Doppler tracking in 
Fig. 1 The angle between the Sun and (a) Vesta and (b) Ceres as viewed from the Earth for the duration of the Dawn mission science phases. As the Sun angle becomes smaller, the Doppler noise increases. The area in red indicates the times when the Sun angle does not satisfy the Doppler accuracy requirement
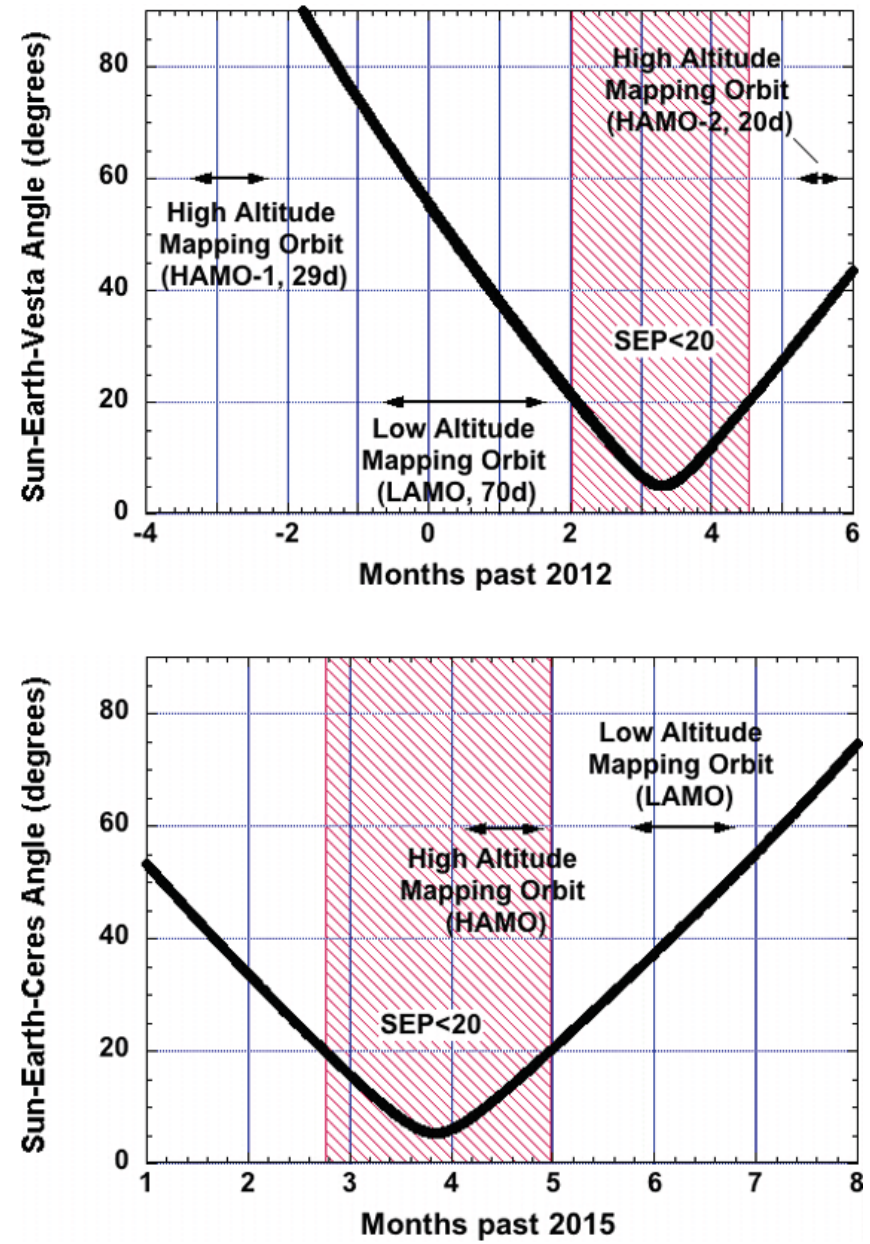

order to accurately construct the spacecraft's motion and small perturbations in its accelerations. In this mode, an uplink signal is derived from the highly stable hydrogen maser atomic clock (frequency reference) at the ground station and transmitted to the spacecraft which, one-way light time later, locks onto it in the transponder and generates a coherently related downlink signal that maintains the phase stability when received back at the ground station. The typical resulting noise in the Doppler observables can reach a data quality of $\sim 0.05 \mathrm{~mm} / \mathrm{s}$ or better at 10 -second integration times. This measure of the gravity science data quality depends on several factors that aggregately constitute the experiment's error budget as detailed in Asmar et al. (2005), including the effects of media through which the radio beam propagates and the standard calibration techniques for those effects. Two error factors, however, are relevant to the planning and execution of the Dawn gravity observations: the angular proximity of the radio beam to the Sun and the received signal-to-noise ratio (SNR).

The first factor, the angle between the Sun and Vesta as viewed from the Earth (see Fig. 1), bounds the duration of the experiment, since the solar plasma significantly increases the Doppler noise at angles under $20^{\circ}$. As an example, Fig. 2 shows how the Doppler accuracy degrades as Sun angle decreases for the X-band Mars Global Surveyor (MGS) and Mars Odyssey missions. The accuracy versus Sun angle is expected to be the same for Dawn. Since the Dawn gravity investigation requires the Doppler accuracy for the mission to be better than $0.15 \mathrm{~mm} / \mathrm{s}$ at 10 -s samples, the tracking coverage for the gravity experiment is to occur for Sun angles $>20^{\circ}$. The history of the Sun angle for all mission phases in 


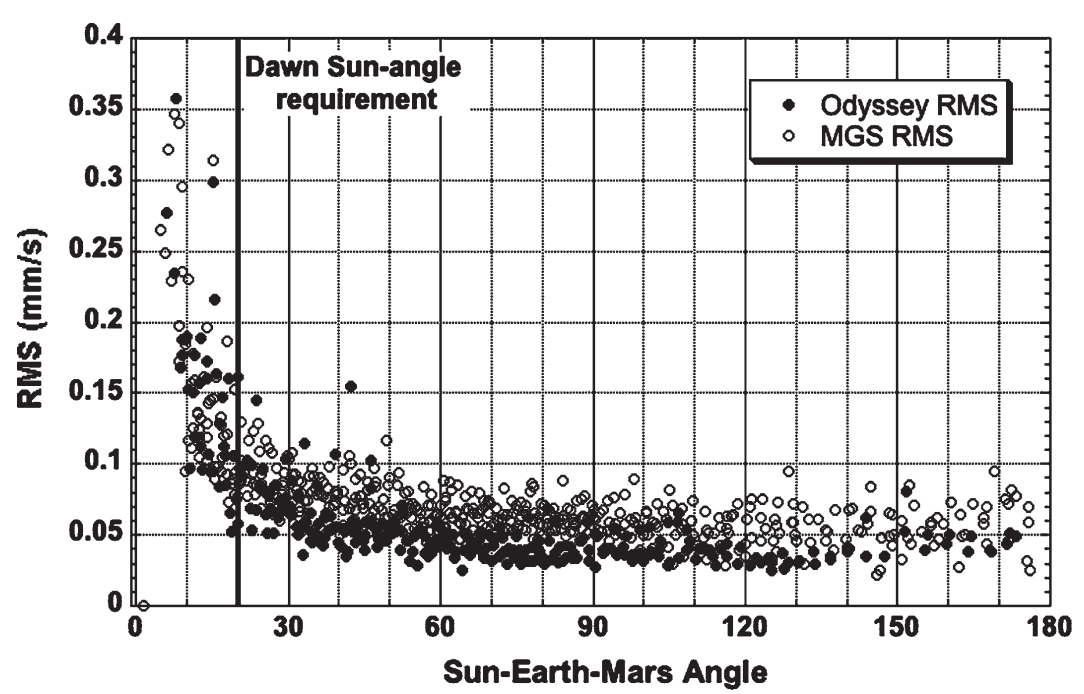

Fig. 2 Doppler noise accuracy for the MGS and Mars Odyssey missions versus Sun angle. Similar accuracy is expected for the Dawn mission

shown in Fig. 1. The Dawn spacecraft will be first placed into a survey orbit around Vesta for 17 days beginning August 10, 2011, and the initial Sun angle is near $150^{\circ}$. After a transition orbit, the first HAMO phase of 30 days begins September 23, 2011, where the sun angle is about $90^{\circ}$. However, the Sun angle decreases to much smaller values for the 70 day LAMO phase that starts December 11, 2011. A second 20-day HAMO phase then begins June 8, 2012 after superior conjunction. With the current mission plan for Ceres, the Sun-EarthCeres angle is below $20^{\circ}$ for the 21 day HAMO orbit from early May to late May 2015, but the Sun angle starts at $35^{\circ}$ in late June 2015 for the LAMO phase and increases thereafter. Note that the actual science phase dates may change due to mission re-plans, but the orbit geometry and tracking schedule will remain similar to those that are assumed in this paper.

The second factor of SNR determines the usability of low gain antennas in the context of other instrument data acquisition that can lead to additional gravity data. Although the Doppler noise does not decrease with higher SNR, there is a minimum level threshold to overcome the phase jitter of the link's thermal noise. For a carrier frequency $f(8435 \mathrm{MHz})$, integration time $T$ (60 s), transponder turn-around ratio $G(880 / 749)$, and transponder carrier loop bandwidth $B$ in $\mathrm{Hz}$, the error contribution $\sigma_{V}$ in units of velocity due to thermal noise is, based on Sniffin et al. (2000):

$$
\sigma_{V}=\frac{c}{2 \sqrt{2} \pi f T} \sqrt{\frac{1}{\rho_{\text {down }}}+\frac{G^{2} B}{\left(P_{C} / N_{0}\right)_{u p}}}
$$

where $\rho_{\text {down }}$ is ratio of carrier to noise power in the downlink receiver loop and $\left(P_{C} / N_{0}\right)_{\text {up }}$ is the uplink carrier power to noise spectral density ratio, $\mathrm{Hz}$ (the latter of which is typically not a limiting factor due to the high power transmitters and gain of the ground stations).

Dawn's transponder is the Small Deep Space Transponder type, common to most NASA deep space missions of the era and proven for excellent performance for Radio Science at the $\sim 3.6 \mathrm{~cm} \mathrm{X}$-band wavelength. The receiver acquires and tracks the uplink carrier via a phaselocked loop and produces a voltage controlled oscillator signal whose phase is coherent with the uplink carrier. For the coherent downlink transmission mode required for gravity and navigation, the exciter derives a coherent X-band carrier from the receiver frequency. When coherency is not achievable (e.g., no uplink from a ground station), the downlink carrier is 
derived from the auxiliary oscillator and the Doppler noise of this internal frequency source is too high to be useful to gravity science.

The telecommunications subsystem is equipped with a traveling-wave tube amplifier (TWTA) that raises the transponder's output signal level to 100 Watts prior to radiating through the antenna in order to meet the telemetry highest bit rate requirement at the furthest distances of the mission. The High Gain Antenna (HGA) is a 1.52-m diameter paraboloid with a nominal downlink bore sight gain of $39.6 \mathrm{dBi}$ (Taylor 2009). It is fixed mounted to the $+\mathrm{X}$ panel of the spacecraft, thus requiring spacecraft pointing to Earth. There are also three Low Gain Antennas (LGA) whose peak gain is $\sim 6 \mathrm{dBi}$, mounted along the $+x,+z$, and $-z$ axes, that sacrifice gain for beam width in order to provide a uniform coverage over various spacecraft orientations for purposes of low data rate communications, especially during engine thrusting, and during safe mode. It is expected that the spacecraft's HGA will be used for the planned gravity observations, providing sufficient SNR in the link to meet the Doppler noise requirements. The LGAs can also be used to provide additional data when the HGA is not pointed to Earth to satisfy the needs of other instruments. To achieve the required minimum $0.15 \mathrm{~mm} / \mathrm{s}$ at $10 \mathrm{~s}$ integration, the received carrier to noise power must be $8.6 \mathrm{~dB}-\mathrm{Hz}$ and $22.5 \mathrm{~dB}-\mathrm{Hz}$ to reach the goal of $0.03 \mathrm{~mm} / \mathrm{s}$. Analysis of Vesta LAMO orbit geometry showed that $16 \mathrm{~dB}-\mathrm{Hz}$ are expected from an LGA to a 34 meter diameter DSN station. An LGA link to a 70-m station provides an additional 6-8 dB thus reaching the goal with sufficient margin.

The nominal amount of tracking for Dawn during the gravity phases of the mission is three 8-h (first 6 weeks) or three 5.5-h (next 4 weeks) passes using the HGA per week, one LGA pass per day using the 70-m DSN antennas, and additional continuous tracking using the LGAs with the DSN 34-m antennas. Expected results are given below for two scenarios-one 5.5-h pass per day and continuous tracking. Actual results are expected to be between the two. The ground track spacing for the HGA plus 70-m LGA tracking satisfies the $30-\mathrm{km}$ and $100-\mathrm{km}$ spacing requirement at Vesta and Ceres, respectively. In addition to Doppler tracking, one to two meter accuracy range data will be acquired, and this is essential to eliminating Vesta or Ceres ephemeris errors from the gravity solution.

Science images will be acquired and used for optical navigation at all mission phases (Polansky et al. 2011). The main optical observables will be landmark vectors from the center of the body to the surface. Landmarks are control points at the center of a local digital elevation model or maplet. A maplet is defined by its control point, its local coordinate system and a local grid, typically $99 \times 99$ pixels. Each grid-point contains the local elevation relative to the control point as well as a relative albedo value.

The details of landmark-based optical navigation for Dawn have been presented elsewhere (Mastrodemos et al. 2011). Here we give a brief summary. Each maplet and its associated landmark vector is constructed as follows: (1) combine images at varying view angles and incidence angles, with a minimum of 3 images needed, (2) from each image set extract a template of a predefined grid scale and size and normally-project it onto the a-priori asteroid surface (a-priori shape model), (3) for each image template construct a brightness model, a step which includes an assumption about the reflectance model of the asteroid, (4) perform a least-squares fit to the brightness model and estimate at each maplet pixel the local slopes and relative albedo, (5) integrate the slopes to construct the heights.

Once a number of maplets have been constructed a global geometric solution is performed (see Fig. 3), that solves for the landmark vector (one per maplet), the camera pointing and the spacecraft-asteroid center relative vector, the latter in a kinematic sense, i.e., on a per-image basis. Subsequently the existing maplets are re-derived, this time starting with better initial conditions of camera pointing and landmark vectors. Additional data types that 
Fig. 3 A simulated Vesta Survey image of the south pole region. The white square circles mark the locations of the landmark vectors that are included in this image

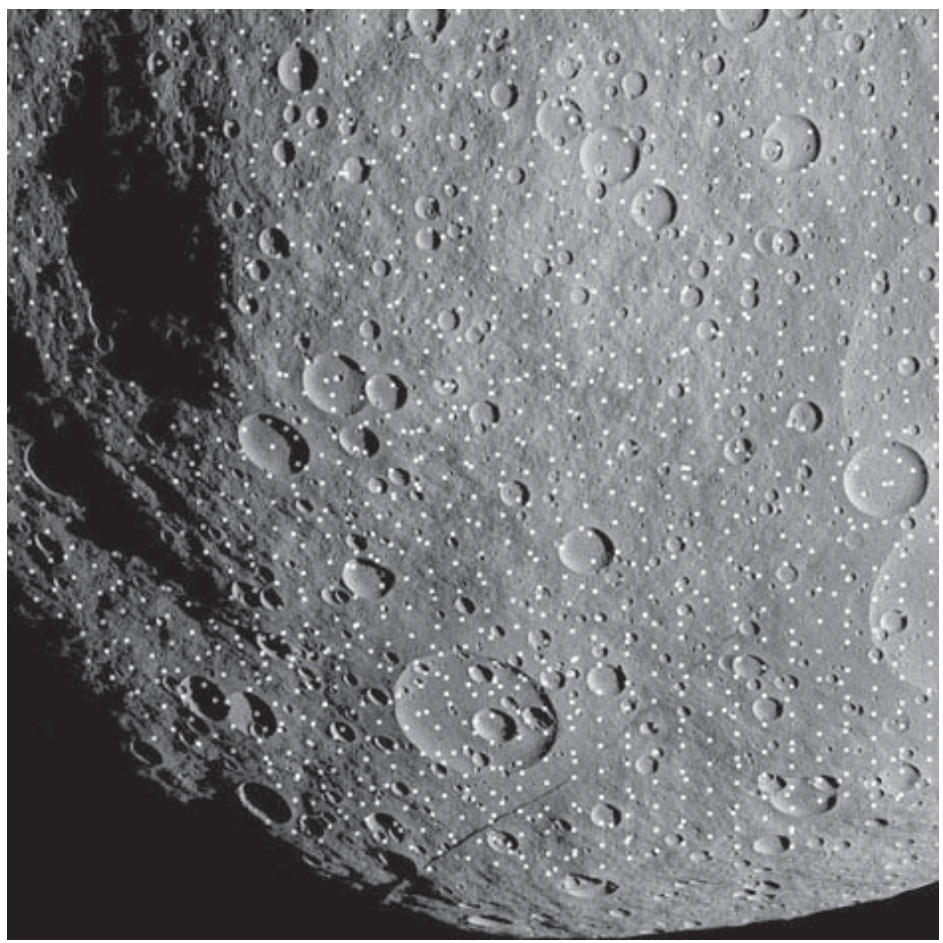

contribute to this solution are the maplet-to-maplet overlap vectors and the limb apparition of maplets. This process of maplet construction, global geometric solution and outlier rejection iterates at least 2, 3 times with each data set, prior to adding new images and/or new maplets.

At every mission phase a network of landmarks that correspond to tightly overlapping maplets is constructed. The landmark network begins with relatively coarse resolution maplets and sparse spacing of landmark vectors from the approach observations and it is subsequently refined at the next mission phase. The optical measurements consist of the image pixel, line location of each landmark and of a Cartesian vector from the center of mass to the surface (landmark vector). Assuming that a number of images, typically 5 or 6, at varying incidence and emission angles are included in each landmark, to first order, both the accuracy of the landmark pixel, line location in the image and the accuracy of the landmark vector will depend on the image resolution and the maplet scale. Typically, at the beginning of each processing phase, the maplet scale is coarser by up to twice that of the image scale and at the end of each processing phase it is comparable to that of the image resolution. If a large number of images, larger than $\sim 10$, has contributed for the construction of each maplet, then the problem of the local topography estimation can become highly over-determined. In this case, as with the Survey observations, maplets up to $1 / 2$ the image scale can be constructed, allowing even higher landmark accuracy, although in this case the scaling may not be linear with maplet resolution.

Typical $1-\sigma$ accuracy estimates at the end of each processing phase are for a post-fit image pixel, line residuals of $\sim 1 / 3$ image pixels and for the landmark vector $\sim 1 / 3$ of the corresponding maplet scale (km/pixel) per Cartesian component. For example, at the last rotational characterization at a range of $\sim 5000 \mathrm{~km}$ we expect pixel, line post-fit residuals of $\sim 150 \mathrm{~m}$ and landmark vector accuracy of $\sim 125-150 \mathrm{~m}$. According to the current mission plan at Vesta the approximate number of images, both for optical navigation and science, and the respective number of landmarks and optical data errors for each mission phase are given in Table 2. Note that the LAMO images are acquired all at a nadir direction and although 
Table 2 Expected number of images and performance for optical landmark tracking for each Dawn mission phase at Vesta

\begin{tabular}{|c|c|c|c|c|c|}
\hline $\begin{array}{l}\text { Mission } \\
\text { Phase }\end{array}$ & $\begin{array}{l}\text { Total \# of images } \\
\text { (mean resolution) }\end{array}$ & $\begin{array}{l}\text { Approximate } \\
\text { total \# of } \\
\text { landmarks }\end{array}$ & $\begin{array}{l}\text { Average \# of } \\
\text { landmarks } \\
\text { per image }\end{array}$ & $\begin{array}{l}\text { Landmark } \\
\text { vector } 1-\sigma \\
\text { Position error }\end{array}$ & $\begin{array}{l}\text { Landmark pixel, } \\
\text { line post-fit } \\
\text { residual in meters }\end{array}$ \\
\hline Survey & $520(280 \mathrm{~m})$ & $\begin{array}{l}12000 \text { at } \\
150 \mathrm{~m} / \text { pixel }\end{array}$ & $>600$ & $140 \mathrm{~m}$ & $140 \mathrm{~m}$ \\
\hline HAMO1 & $2400(60 \mathrm{~m})$ & $\begin{array}{l}120000 \text { at } \\
50 \mathrm{~m} / \text { pixel }\end{array}$ & 50 & $40 \mathrm{~m}$ & $40 \mathrm{~m}$ \\
\hline $\begin{array}{l}\text { HAMO1+ } \\
\text { HAMO2 }\end{array}$ & $3900(60 \mathrm{~m})$ & $\begin{array}{l}120000 \text { at } \\
50 \mathrm{~m} / \text { pixel }\end{array}$ & 30 & $40 \mathrm{~m}$ & $40 \mathrm{~m}$ \\
\hline LAMO & $2000(25 \mathrm{~m})$ & $\begin{array}{l}120000 \text { at } \\
50 \mathrm{~m} / \text { pixel }\end{array}$ & 30 & $40 \mathrm{~m}$ & $40 \mathrm{~m}$ \\
\hline
\end{tabular}

they allow an almost complete coverage of the asteroid surface, they are regarded more as supplementary to HAMO images, rather than a complete data set in its own right.

\section{Spacecraft Models}

The major features of the Dawn spacecraft including the spacecraft coordinate frame, science instruments, and antenna locations are shown in Fig. 4. The high aspect-ratio solar panels are a distinctive feature of the Dawn spacecraft and represent a larger surface area $\left(36.5 \mathrm{~m}^{2}, \sim 50 \%\right.$ increase in area to mass ratio) than other spacecraft such as MRO, MGS, Odyssey and NEAR because of the power required for the ion engines. The central ion engine is located along the $-z$ thrust direction, and the other two ion engines are located in the $x-z$ plane and are canted about $\pm 40^{\circ}$ from the $-z$ direction. No gravity data is processed when the ion engines are in operation. The solar panels, which can rotate along the $y$-axis, always points near normal to the Sun direction during all phases of the mission. The spacecraft attitude is determined by the power-steering algorithm, which computes the attitude given a spacecraft body-fixed aim vector (e.g. instrument deck or $+z$ direction for science observations and $+x$ direction for HGA tracking) and a target vector (e.g., Vesta for science observations and Earth for HGA tracking). The body-fixed aim vector is first aligned with the nadir or Earth pointed target vector, during most of science phases, and the spacecraft rotates about the aim vector axis and finds a solution which in order of priority puts first the $-x$-axis (Visible InfraRed Spectrometer or VIR, radiators, and star trackers) or second the $+z$-axes (Gamma Ray and Neutron Detector or GRaND, Framing Camera, and gyros) away from the Sun. If multiple solutions exist, the solution with the least angular displacement is selected. The spacecraft attitude control subsystem provides attitude determination and control during all mission phases and consists of the following elements: two star trackers looking in the $-x$ direction, an inertial reference unit and four reaction wheel assemblies. The latter are required to maintain stability in the spacecraft motion to minimize non-gravitation acceleration, which would be another noise factor for gravity science. The quaternions for the spacecraft attitude and solar array orientation with respect to the spacecraft frame will be obtained from the Planetary Data System (PDS) archive (Semenov, private communication, 2010).

During gravity science phases, a body-fixed high-gain antenna (HGA) will be used for tracking when it's available; otherwise, low-gain antennas (LGAs) will be used. When the 
Fig. 4 The Dawn spacecraft with location of science instruments and antennas. The spacecraft coordinate frame is shown; its origin is at the baseplate of the central ion engine

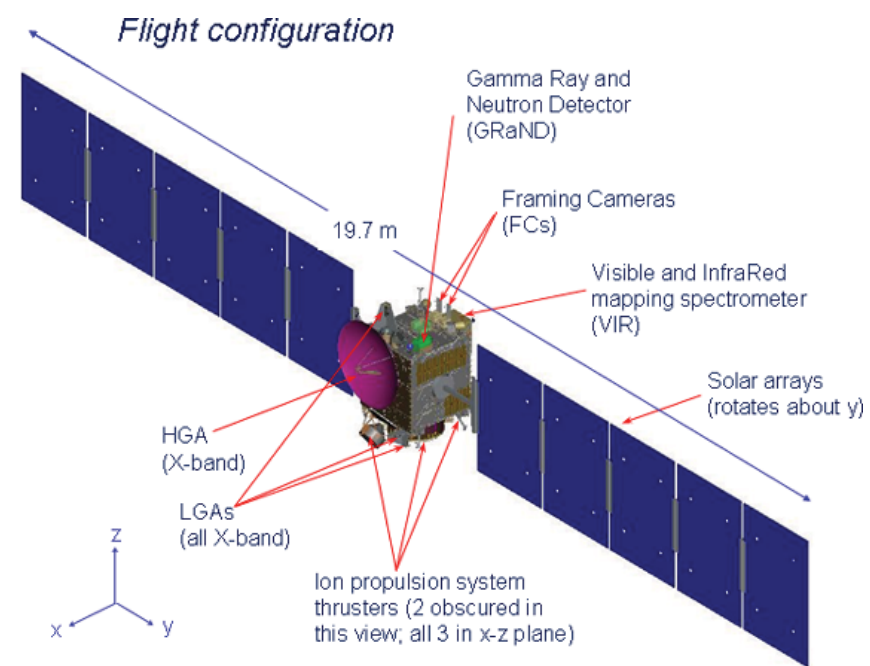

HGA is in use, the HGA will be pointing toward the Earth, and phase center location with respect to the spacecraft center-of-mass remains constant in the Earth direction. Thus, the spacecraft attitude is not needed in the HGA Doppler measurement model. However, when LGAs are used, the spacecraft will be slowly changing its attitude with respect to Earth to maintain nadir point, which requires the LGA motion with respect to the center of mass in the Doppler measurement model. Note that there are three LGAs as shown in Fig. 4.

Figure 5 shows the current reference trajectory designs for HAMO and LAMO at Vesta and Ceres. The center bodies are drawn to scale to reflect the orbit sizes (based on current shape estimates). The major forces acting on the spacecraft are the accelerations due to gravity and solar radiation pressure. Figures 6 and 7 show the expected accelerations during HAMO and LAMO mission phases. Note that CBP refers to the central body point-mass acceleration, OBL refers to the higher-order gravity acceleration (non-point mass acceleration), and SRP refers to the acceleration due to solar radiation pressure. The trajectory was integrated using JPL's DE421 ephemerides for positions of the planets.

Since Vesta is a differentiated body (e.g., Keil 2002), a simple three-layered model was used as the nominal field for generation of the reference orbit (similar to models tested by Tricarico and Sykes 2010). The 15th spherical harmonic degree gravity field model consists of a spherical $150-\mathrm{km}$ radial core of density $5.8 \mathrm{gm} / \mathrm{cm}^{3}$ at the center-of-figure, an ellipsoidal mantle $\left(250 \times 250 \times 227-\mathrm{km}\right.$ and $\left.3.0 \mathrm{gm} / \mathrm{cm}^{3}\right)$ and a crust $\left(2.7 \mathrm{gm} / \mathrm{cm}^{3}\right)$ from the shape model (Thomas et al. 1997) with variable thickness (zero near the southern crater and $\sim 20-40-\mathrm{km}$ elsewhere). Multiple models were tested and the accuracy of the gravity field that will be determined at Vesta is not that sensitive to the nominal field chosen for the simulation (various core densities and location or even a uniform density Vesta). The gravity acceleration for Vesta in Fig. 6 uses the nominal 3-layer model. The accelerations from an assumed uniform density gravity field model are about $20 \%$ higher and represent a probable upper bound of all various internal models. The Ceres gravity acceleration used a 20th degree gravity field which was sampled based on a Kaula rule, i.e., $K_{\text {Ceres }}=0.0086$ (see below).

The solar pressure acceleration is computed using the appropriate specular and diffuse reflectivity coefficients for a multiple flat-plate box-wing spacecraft model. Although the acceleration due to solar radiation pressure is several orders of magnitude smaller than the gravity acceleration (and 50\% higher than typical due to a larger solar panel), it is one of the limiting error sources for high-precision determination of the gravity field and pole location. 


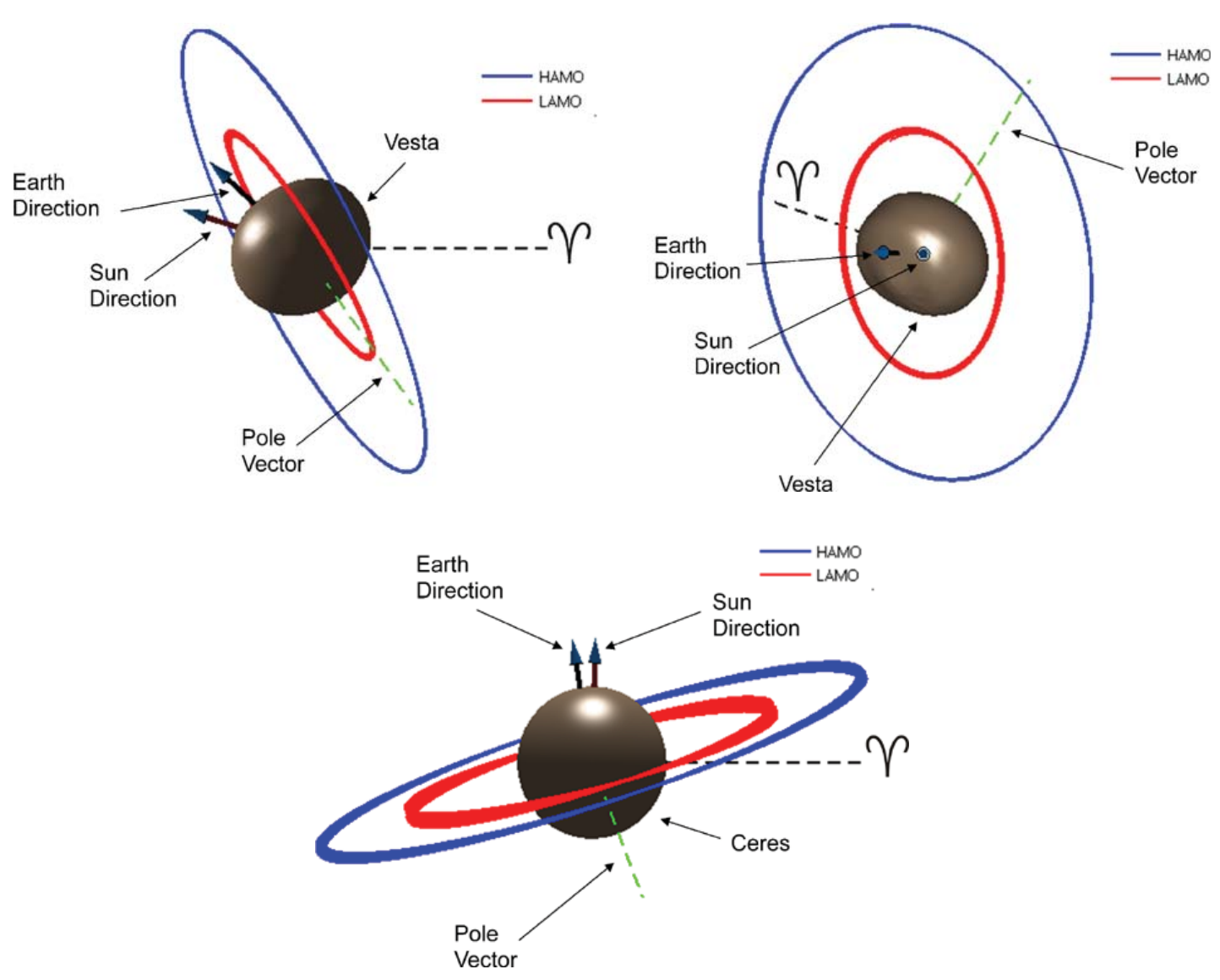

Fig. 5 Views of the HAMO and LAMO orbits at (a) Vesta as viewed from the ecliptic north pole and Sun and (b) Ceres as seen from the ecliptic north pole. The directions of the Sun and Earth are also shown

Another important force that will affect the gravity science is the angular momentum dump (AMD), which stabilizes the on-board wheel speeds using hydrazine thrusters. The largest expected magnitude of AMDs during HAMO and LAMO phases is near $10 \mathrm{~mm} / \mathrm{s}$ with a duration of 10-20 minutes. Also note that AMD magnitude will be higher during LAMO than HAMO since the gravity gradient force increases as the orbit radius decreases. The magnitudes are not as important as the AMD frequency since corrections to the AMD amplitudes will be estimated as a delta-V maneuver. The longer the duration between the AMDs, the better the gravity information in the data arc. The current science performance presented below assumes an AMD maneuver every two days for both HAMO and LAMO (close to the planned three times per week for LAMO but more conservative for the expected once per week in HAMO). During the LAMO phases, the current nominal plan assumes that there will be an orbit maintenance maneuver (OMM) every 7 days.

\section{Gravity Determination Technique}

The gravity fields of Vesta and Ceres will be determined through the gravitational perturbations of the orbit of the spacecraft. The technique and software (Orbit Determination Program or ODP, Moyer 1971) are the same as that used for previous planetary and small body gravity analysis (e.g., Konopliv et al. 2011 for Mars and Konopliv et al. 2002 for Eros). The equations of motion and variational partials for the estimated parameters are numerically integrated (variable order Adams method, Krogh 1973) for the nominal motion 

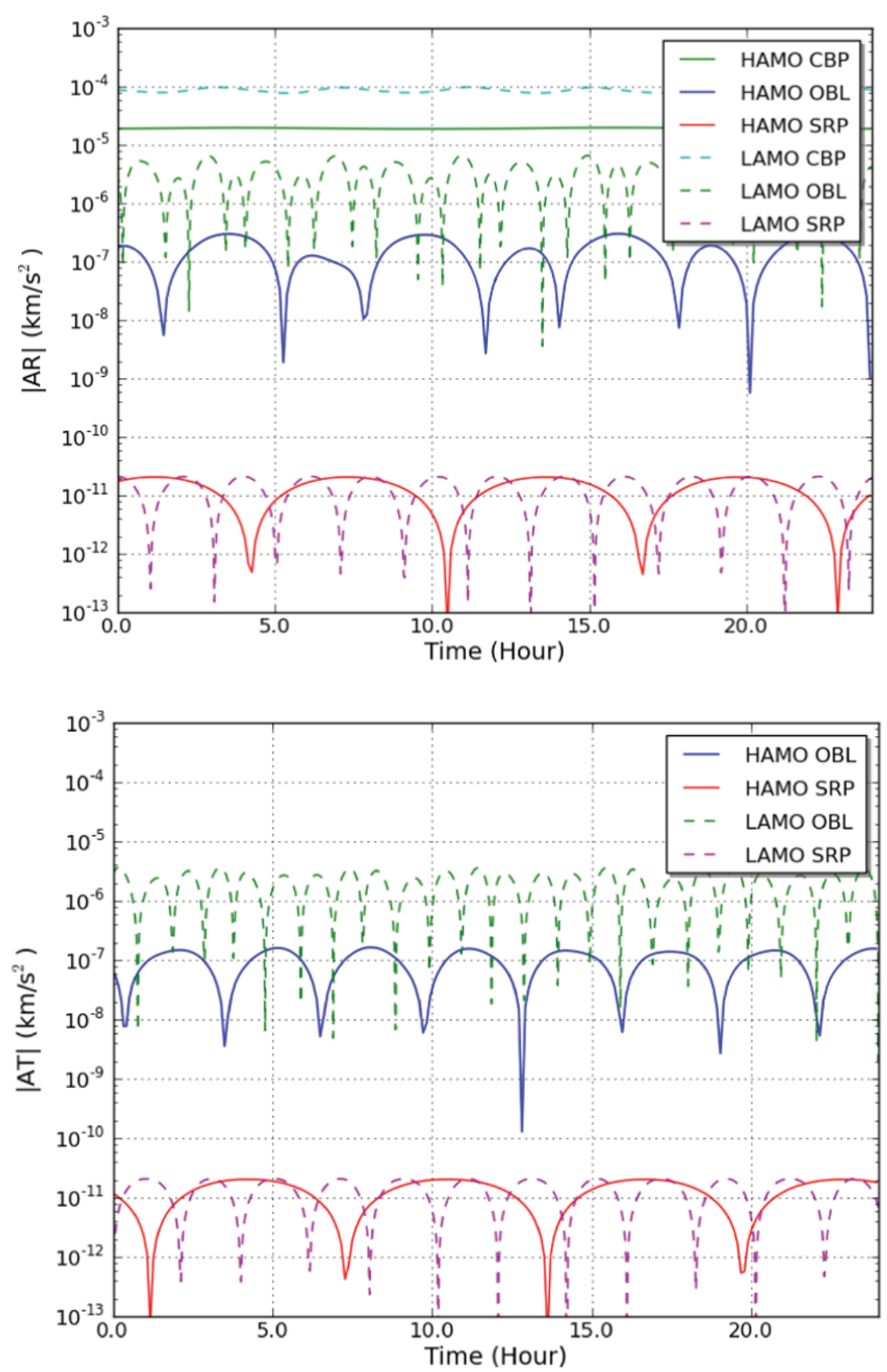

Fig. 6 The accelerations on the Dawn spacecraft at Vesta due to the central two-body point mass (CBP), the gravitational oblateness (OBL) and solar radiation pressure (SRP). The constant density Vesta gravity model was used to compute the oblateness acceleration. The accelerations are given the radial $(R)$, along the velocity $(T)$ and normal to the orbit plane $(N)$ directions

of the spacecraft in the International Celestial Reference Frame (ICRF, within $<100$ mas of Earth Mean Equator of 2000; Folkner et al. 1994). All forces acting on the spacecraft are accounted for including gravity, solar radiation pressure, thrusting (mostly for despin of the angular momentum wheels), and point mass contributions from the Sun and planets. The DSN Doppler and landmark observations are then processed (Moyer 2000) to obtain the linearized observation equations (e.g., Tapley et al. 2004). 


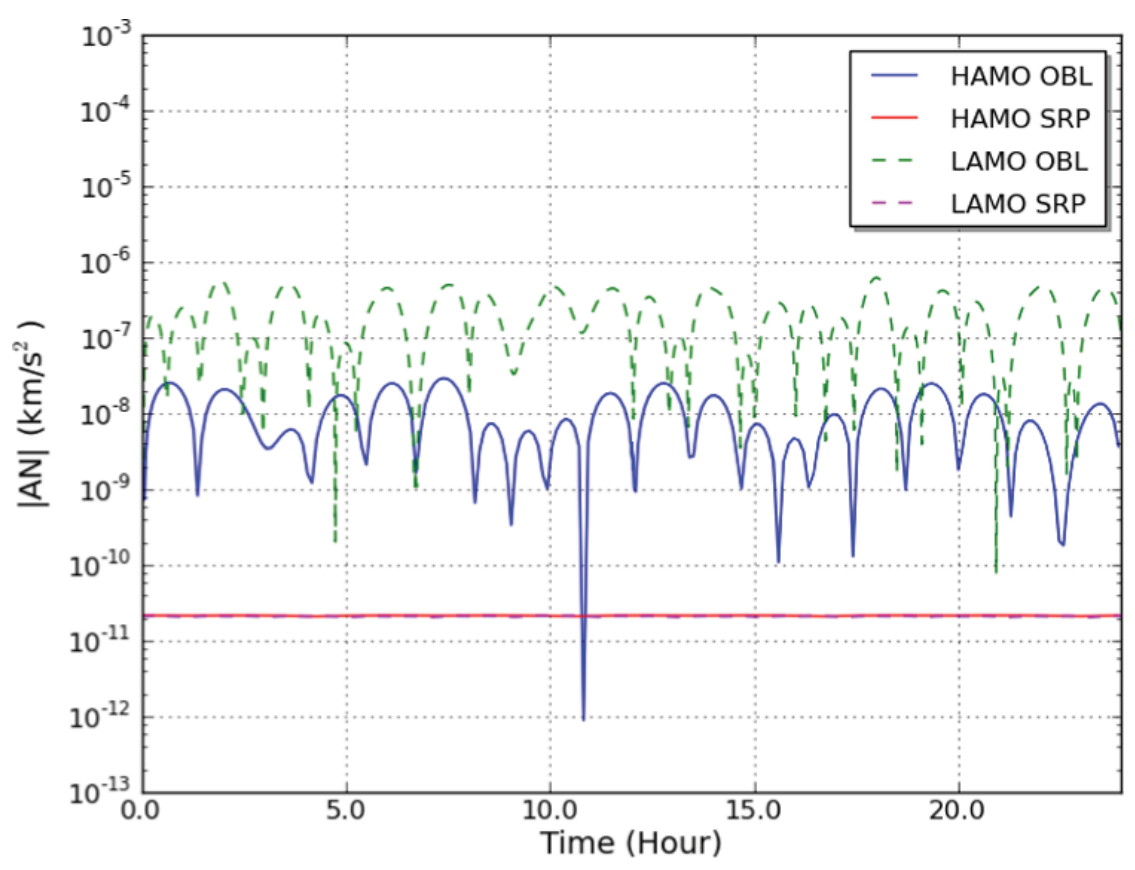

Fig. 6 (Continued)

The spacecraft state and other parameters are estimated using a square root information weighted least squares filter (or SRIF, see Lawson and Hanson 1995, and Bierman 1977). The observations are divided into time spans that we call data arcs. For the Dawn mission we expect arc length to be about 4 days for LAMO, but longer arc lengths will be investigated to the point where gravity results are not degraded by modeled non-gravitational forces. The estimated parameters consist of local parameters specific to the data arc such as spacecraft position and velocity, velocity corrections for angular momentum wheel despins, and solar pressure coefficients and global parameters common to all data arcs such as gravity, pole location and rotation rate. The global parameters are determined from merging the global SRIF data array from each arc similar in concept to the partitioned normal matrix method (Kaula 1966).

The gravitational potentials of Vesta and Ceres are modeled by a spherical harmonic expansion in the body-fixed reference frame with normalized coefficients $\left(\bar{C}_{n m}, \bar{S}_{n m}\right)$ that is given by (e.g., Heiskanen and Moritz 1967; Kaula 1966)

$$
U=\frac{G M}{r}+\frac{G M}{r} \sum_{n=1}^{\infty} \sum_{m=0}^{n}\left(\frac{R_{e}}{r}\right)^{n} \bar{P}_{n m}\left(\sin \phi_{l a t}\right)\left[\bar{C}_{n m} \cos (m \lambda)+\bar{S}_{n m} \sin (m \lambda)\right],
$$

where $G M$ is the gravitational constant times the mass of the central body, $n$ is the degree, $m$ is the order, $\bar{P}_{n m}$ are the fully normalized associated Legendre polynomials, $R_{e}$ is the reference radius of the body (265-km for Vesta, $467-\mathrm{km}$ for Ceres), $\phi_{\text {lat }}$ is the latitude, and $\lambda$ is the longitude. The gravity coefficients are normalized such that the integral of the harmonic squared equals the area of a unit sphere, and are related to the unnormalized coefficients as (Kaula 1966; Lambeck 1988)

$$
\left(\begin{array}{c}
C_{n m} \\
S_{n m}
\end{array}\right)=\left[\frac{(n-m) !(2 n+1)\left(2-\delta_{0 m}\right)}{(n+m) !}\right]^{1 / 2}\left(\begin{array}{c}
\bar{C}_{n m} \\
\bar{S}_{n m}
\end{array}\right) .
$$



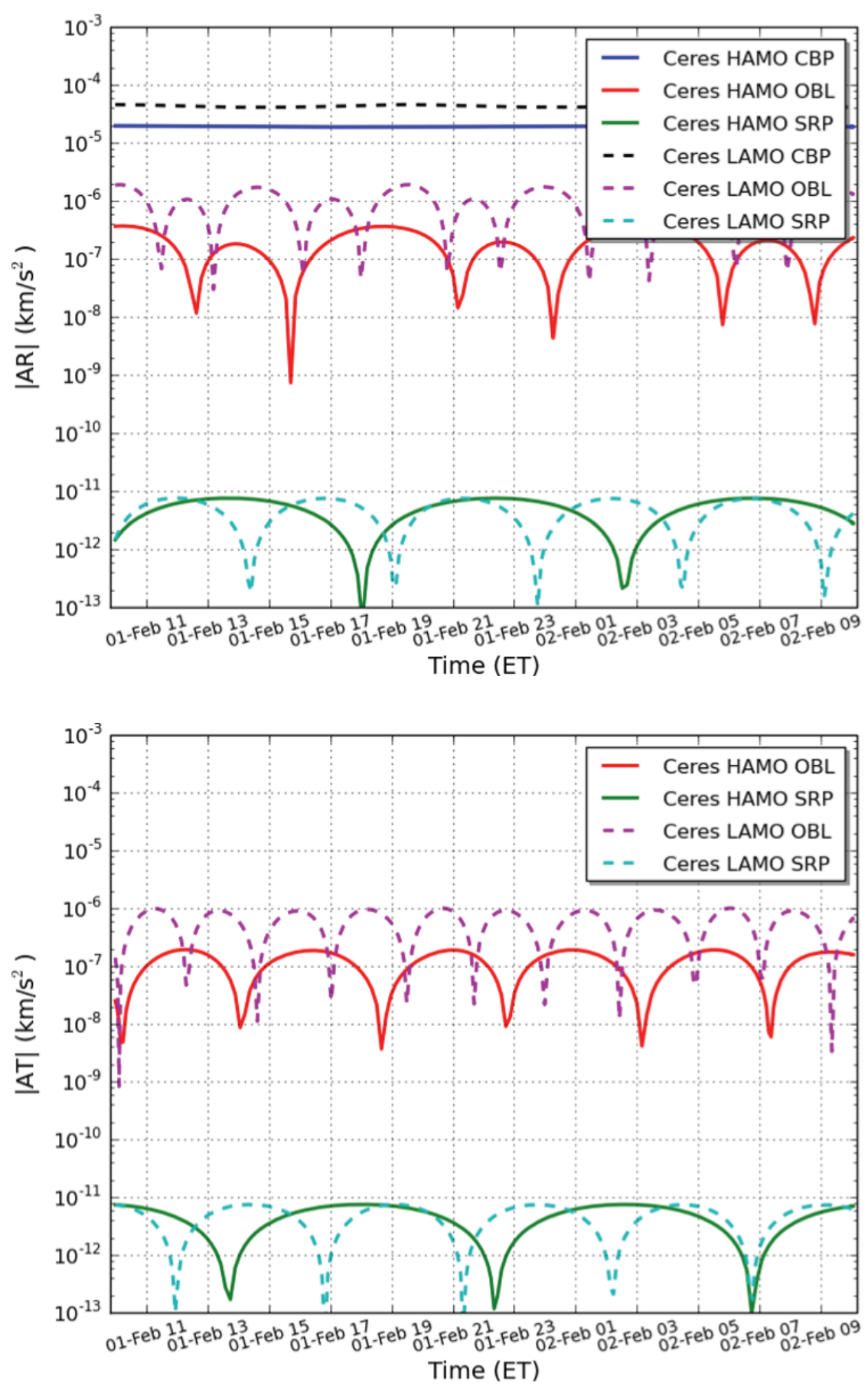

Fig. 7 The accelerations on the Dawn spacecraft at Ceres due to the central two-body point mass (CBP), the gravitational oblateness (OBL) and solar radiation pressure (SRP). A sample 20th degree harmonic field based upon the expected Kaula power rule was used for the oblateness acceleration. The accelerations are given the radial (R), along the velocity $(\mathrm{T})$ and normal to the orbit plane $(\mathrm{N})$ directions

The degree one coefficients are zero, since the origin of the coordinate system is chosen to be the center of mass. The zonal coefficients are given by $\bar{J}_{n}=-\bar{C}_{n 0}$.

The RMS magnitude spectrum of the gravity field tends to follow a power law of the form (Kaula 1966) 


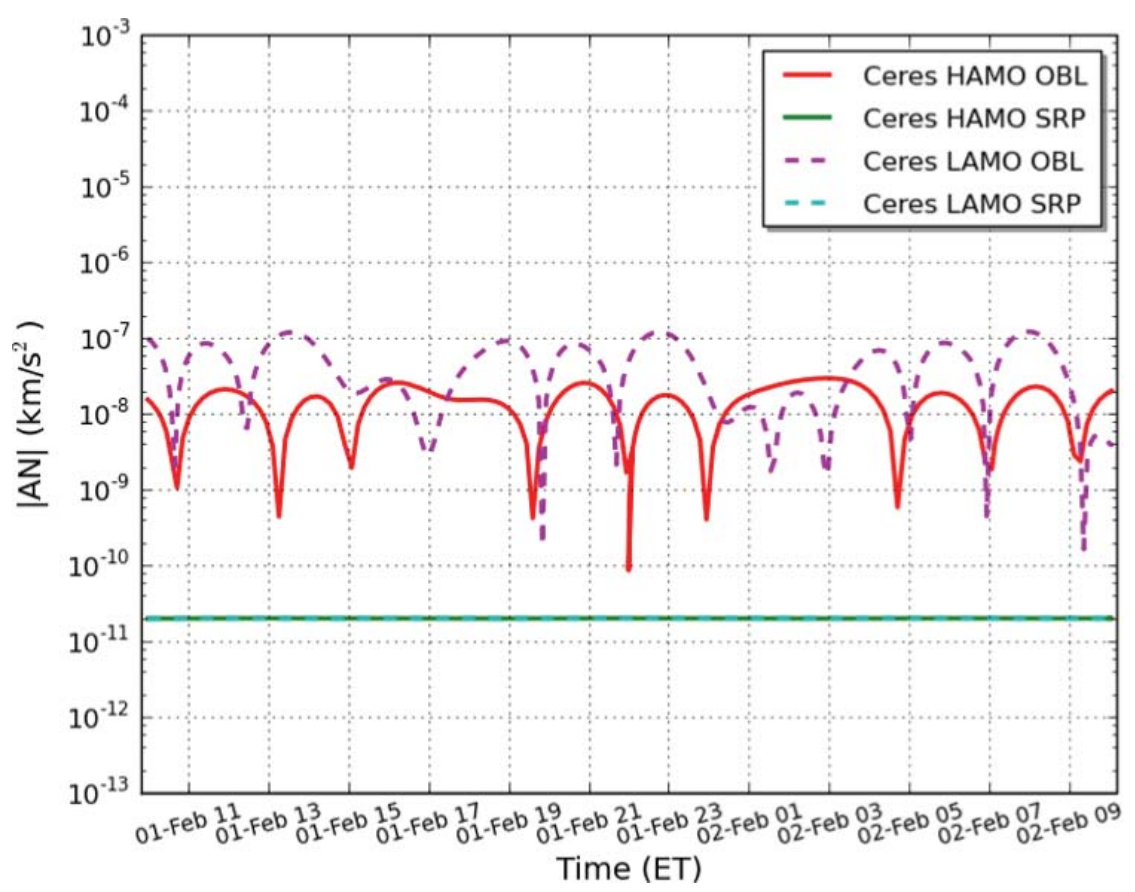

Fig. 7 (Continued)

$$
M_{n}=\sqrt{\frac{\sum_{m=0}^{n}\left(\bar{C}_{n m}^{2}+\bar{S}_{n m}^{2}\right)}{2 n+1}} \approx \frac{K}{n^{2}}
$$

where the constant $K$ is $\sim 10^{-5}$ for the Earth (values for Vesta and Ceres are discussed below). A constraint of this form is often applied to the gravity coefficients in order to limit excessive power in the poorly determined coefficients of higher degree. The coefficients are biased to zero with an uncertainty given by the power law. To avoid suppressing gravity information in the better-known coefficients, the constraint is sometimes only applied to parts of the spectrum where $M_{n}$ exceeds the power law.

Combining spherical harmonic coefficients from the anticipated shape models for Vesta and Ceres (Raymond et al. 2011b) will allow long-wavelength estimates of interior structure (cf. McCord et al. 2011; Zuber et al. 2011) posed in terms of crustal and elastic thicknesses or mantle density variations (Neumann et al. 1996; Wieczorek 2007).

\section{Moments of Inertia from Gravity, Wobble and Obliquity}

In addition to determining the mean densities of Ceres and Vesta, the Dawn mission may be able to obtain estimates of their moments of inertia. Together, the mean density and mean moment of inertia provide two integral constraints on the radial density profile, and allow better determination of the internal density structure (Bills and Rubincam 1995).

There is a close relationship between the inertia tensor of a body and the degree two coefficients in a spherical harmonic expansion of the gravitational potential (Soler 1984). However, there are only 5 degree two coefficients and 6 independent terms in the inertia tensor. Thus, even if we have a highly accurate gravity field, we will still lack sufficient information to directly determine the moments of inertia. We now discuss three possible means of estimating the remaining constraint. 


\subsection{Moment from gravity}

If the coordinate axes are chosen to coincide with principal inertial axes, the inertia tensor diagonalizes, three of the potential coefficients vanish and the remaining two unnormalized gravity coefficients, which we denote $J_{2}$ and $C_{2,2}$, have a simple relationship to the principal moments $A<B<C$

$$
\left\lfloor\begin{array}{c}
J_{2} \\
C_{2,2}
\end{array}\right\rfloor M R_{e}^{2}=\left\lfloor\begin{array}{c}
C-(A+B) / 2 \\
(B-A) / 4
\end{array}\right\rfloor
$$

where $M$ and $R$ are the mass and mean radius of the body, respectively.

If a rotating body is in hydrostatic equilibrium, there is a unique relationship between the imposed rotational potential and the induced gravitational potential. The mean moment of inertia

$$
I=(A+B+C) / 3
$$

is not changed by tidal or rotational deformations (Rochester and Smylie 1974), but the principal moments are individually changed. In much of what follows, it will be convenient to use dimensionless versions of the principal and mean moments of inertia, which are given by

$$
\left[\begin{array}{l}
a \\
b \\
c \\
i
\end{array}\right]=\left[\begin{array}{l}
A \\
B \\
C \\
I
\end{array}\right] / M R^{2}
$$

We can write the rotational perturbation as (Bills 1995)

$$
\left\lfloor\begin{array}{l}
A \\
B \\
C
\end{array}\right\rfloor=I+\left\lfloor\begin{array}{l}
-1 \\
-1 \\
+2
\end{array}\right\rfloor \frac{k \omega^{2} R_{e}^{5}}{9 G}
$$

where $\omega$ is the rotation rate, $G$ is the gravitational constant, and $k$ is a secular Love number (Munk and MacDonald 1960). The corresponding values of the degree two gravitational potential coefficients are

$$
\left[\begin{array}{c}
J_{2} \\
C_{2,2}
\end{array}\right]=\left[\begin{array}{l}
1 \\
0
\end{array}\right] \frac{k \omega^{2} R_{e}^{3}}{3 G M}
$$

Measurement of $J_{2}$ will provide an estimate of the secular Love number, and the ratio of $C_{2,2} / J_{2}$ will provide an estimate of the proximity to hydrostatic equilibrium. If the hydrostatic assumption is justified, the secular Love number can be used to estimate the polar moment of inertia from the Radau-Darwin relation (Radau 1885; Darwin 1899)

$$
c \equiv \frac{C}{M R_{e}^{2}}=\frac{2}{3}\left(1-\frac{2}{5} \sqrt{\frac{4-k}{1+k}}\right)
$$

This relation holds exactly only for uniform density fluids, but is a reasonable approximation for radially stratified bodies (Nakiboglu 1982).

Current estimates of the moments of inertia of the Galilean satellites of Jupiter all depend upon this approximation (Hubbard and Anderson 1978; Anderson et al. 1996, 1998a, 1998b, 2001). There are two primary differences between those cases and the Dawn encounters with Ceres and Vesta. The Galileo estimates are all derived from multiple fly-by opportunities, 
rather than going into orbit about the bodies of interest. Also, since the Galilean satellites are all in synchronous rotation, they have appreciable tidal contributions, which add to the $J_{2}$ values, and make the expected $C_{2,2}$ values non-zero.

\subsection{Moment from wobble}

If the spin pole of either Ceres or Vesta exhibits a free wobble, or misalignment with the axis of greatest inertia, that would be informative in two ways. First, it would suggest a very recent excitation event, and second, it would provide a means of estimating the moment of inertia.

The rotational configuration of lowest energy, for a given angular momentum, consists of steady rotation about the axis of greatest moment of inertia. If the body is rotating about another axis, it will exhibit a wobble. That is, the instantaneous rotation vector does not maintain a fixed orientation relative to the body. The free wobble angular rate $w$ is, like the spin pole precession rate, also determined by the spin rate and the moments of inertia. It is given by (Smith and Dahlen 1981)

$$
w=\omega \sqrt{\left(\frac{C-A}{B}\right)\left(\frac{C-B}{A}\right)}
$$

Bills and Nimmo (2011) have estimated wobble periods of 5.5 and 1.2 days for Ceres and Vesta, respectively. If the wobble period could be accurately determined, it would provide one additional constraint, and thereby allow the moments of inertia to be estimated.

There have been numerous analyses of wobble damping, as applied to asteroids and satellites (Burns and Safronov 1973; Harris 1994; Black et al. 1999; Sharma et al. 2005; Samarasinha 2008). The basic idea is that the body is deformed, in response to the rotational potential, and that part of this rotational bulge follows the instantaneous equator as it moves over the surface of the body. The continuing deformation dissipates energy and drives the body toward the lowest energy state of principal axis rotation. Bills and Nimmo (2011) estimate wobble damping times for Ceres and Vesta of 440 and 120 years, respectively. Unless these estimates were based on assumptions that are overly simplistic, or there has been a very recent excitation event, a wobble would not be expected to be observed.

\subsection{Moment from obliquity}

If, as expected, the spin poles of Ceres and Vesta are fully damped, then measurements of their obliquities will also provide information about their moments of inertia. It would also provide information about the amount of dissipation within these bodies. This approach has recently been applied to Titan (Bills and Nimmo 2008) and more recently Ceres and Vesta (Bills and Nimmo 2011).

The moments of inertia are most directly linked to rotational dynamics and the standard astronomical estimates of moments of inertia arise from determination of spin pole precession rates. That is how the moments of inertia of Earth (Williams 1994) and Mars (Folkner et al. 1997) are known. However, the expected precession rates for Ceres and Vesta are small (few arcsec/year) and there is little hope of directly determining precession during the short visits by Dawn.

An alternative exists, which is that, if the spin pole is fully damped, then its instantaneous orientation, relative to the orbit pole, will be diagnostic of the precession rate. The Moon is a classic example of such a body. The orbit pole precesses about the ecliptic pole, and 
the spin pole precesses about the orbit pole. However, due to the achievement of a steady, damped configuration, the spin pole, orbit pole, and ecliptic pole remain coplanar. Such a configuration is now known as a Cassini state (Colombo 1966; Peale 1969; Henrard and Murigande 1987).

The condition for this co-planar precession, in steadily precessing nearly circular orbits, can be written as (Colombo 1966)

$$
2 \beta \sin [\varepsilon]+\alpha \sin [2(i+\varepsilon)]=0
$$

where $i$ is the inclination of the orbit pole to the invariable pole, $\varepsilon$ is the obliquity or separation of spin and orbit pole. The parameter $\beta$ is the rate of orbit plane precession, and $\alpha$ is a spin pole precession rate. It is determined by the moments of inertia of the body, and the relative rates of orbital motion $n$ and spin $s$. It has explicit form

$$
\alpha=\frac{3}{2} \frac{n^{2}}{s}\left(\frac{C-(A+B) / 2}{C}\right)=\frac{3}{2} \frac{n^{2}}{s}\left(\frac{3 J_{2}}{2 J_{2}+3 i}\right)
$$

When the obliquity $\varepsilon$ and gravity coefficients $J_{2}$ and $C_{2,2}$ are all known, the constraint equation (12) can be solved for the polar moment of inertia, without any assumption of hydrostatic equilibrium. However, it does depend upon an assumption that the spin pole is damped.

How likely is it that the spin poles of Ceres and Vesta are damped? For small asteroids, non-gravitational effects can have appreciable influence on rotation rate and direction. For example, many members of the Koronis family of asteroids have non-random spin rates and directions (Slivan 2002; Slivan et al. 2003, 2008), counter to the expectation for purely collisional evolution. This quasi-alignment has been attributed to thermal torques (Vokrouhlicky et al. 2003, 2006; Bottke et al. 2006; Scheeres and Mirrahimi 2008).

For larger and more nearly spherical bodies like Ceres and Vesta, the mechanisms of excitation and damping are less clear. If the near-surface of Ceres is ice-rich (Thomas et al. 2005), its viscoelastic relaxation (Maxwell) time will be short, perhaps even comparable to the rotation period, resulting in enhanced dissipation. Peale (2005) has argued that, for Mercury, where tidal damping is reasonably effective, the dominant obliquity damping mechanism is likely viscous core-mantle coupling. If large asteroids have (or had) fluid regions within them, their spin poles could be fully damped. It has been argued that at least one large asteroid, the Angrite parent body, possessed a fluid core early in its history (Weiss et al. 2008).

One means of testing for an un-damped obliquity would be to look for a free wobble, or non-principal axis rotation (Munk and MacDonald 1960; Lambeck 1980; Chen et al. 2009). The wobble period is short compared to the spin pole precession period, and thus the wobble damping time is short compared to the obliquity damping time. A hypothetical excitation event, such as a relatively recent impact (Peale 1976), could have produced both a free wobble and a free obliquity. If no wobble is found, it would be permissive of a damped obliquity. However, since the obliquity damping time is expected to be much longer, absence of a wobble is no guarantee of a fully damped obliquity. If, on the other hand, an un-damped wobble is found, it would strongly suggest recent excitation. While such a wobble would presumably indicate that the obliquity is not fully damped, measuring the wobble period would provide an alternative way of determining the moment of inertia.

Another means of testing the hypothesis of a damped spin pole is to examine the azimuthal orientation of the spin pole, relative to the orbit pole. As is shown in Bills and Nimmo (2011), if the spin pole is fully damped, its present orientation is fully specified by 
a single parameter, which is the spin pole precession rate. If the observed spin pole does not lie on the curve specified by this model, that would imply there is still a finite free term in the spin pole motion, and the spin pole is not fully damped.

\section{Expected Gravity Science Results at Vesta}

Although there is no direct a priori information for the Vesta gravity field, the expected plausible range of possible gravity fields (e.g., Tricarico and Sykes 2010) can be inferred from the Vesta shape model as determined by the Hubble images (Thomas et al. 1997) and the scaling of the gravity coefficients to an expected power law $K_{V e s t a} / n^{2}$ (Kaula 1966). Figure 8 shows the gravity field without $J_{2}$ on the surface of a sphere (290-km or the maximum equatorial radius to assure convergence near the Brillouin sphere) assuming our 3-layer model for Vesta. The range in surface acceleration is $\sim 2450 \mathrm{mGals}$. The equivalent range from a uniform density model is $\sim 30 \%$ greater.

Lack of convergence within the Brillouin sphere (the smallest sphere that encloses Vesta) is a limitation of the spherical harmonic formulation. A map of the gravity near the surface of Vesta requires alternate representations of the gravity potential such as ellipsoidal harmonics (Garmier and Barriot 2001, and Garmier et al. 2002) that we will investigate as well. The convergence of the spherical harmonics is tested in similar fashion to Garmier and Barriot (2001). The acceleration on an ellipsoid near the Vesta surface was computed using a uniform density polyhedral gravity model (Werner and Scheeres 1997) and the equivalent spherical harmonic representation. For a 20th degree harmonic field and radii of 265-km or greater (or between latitudes of $\pm 50^{\circ}$ or $\sim 75 \%$ of the surface), the RMS difference was 13 mgals ( $<1 \%$ of Fig. 8 ) and a maximum of $\sim 170$ mgals (or $8 \%$ ). However, gravity differences were substantially greater ( $\sim 700$ mgals) near the poles with a radius of $243-\mathrm{km}$ and require the ellipsoidal harmonic representation.

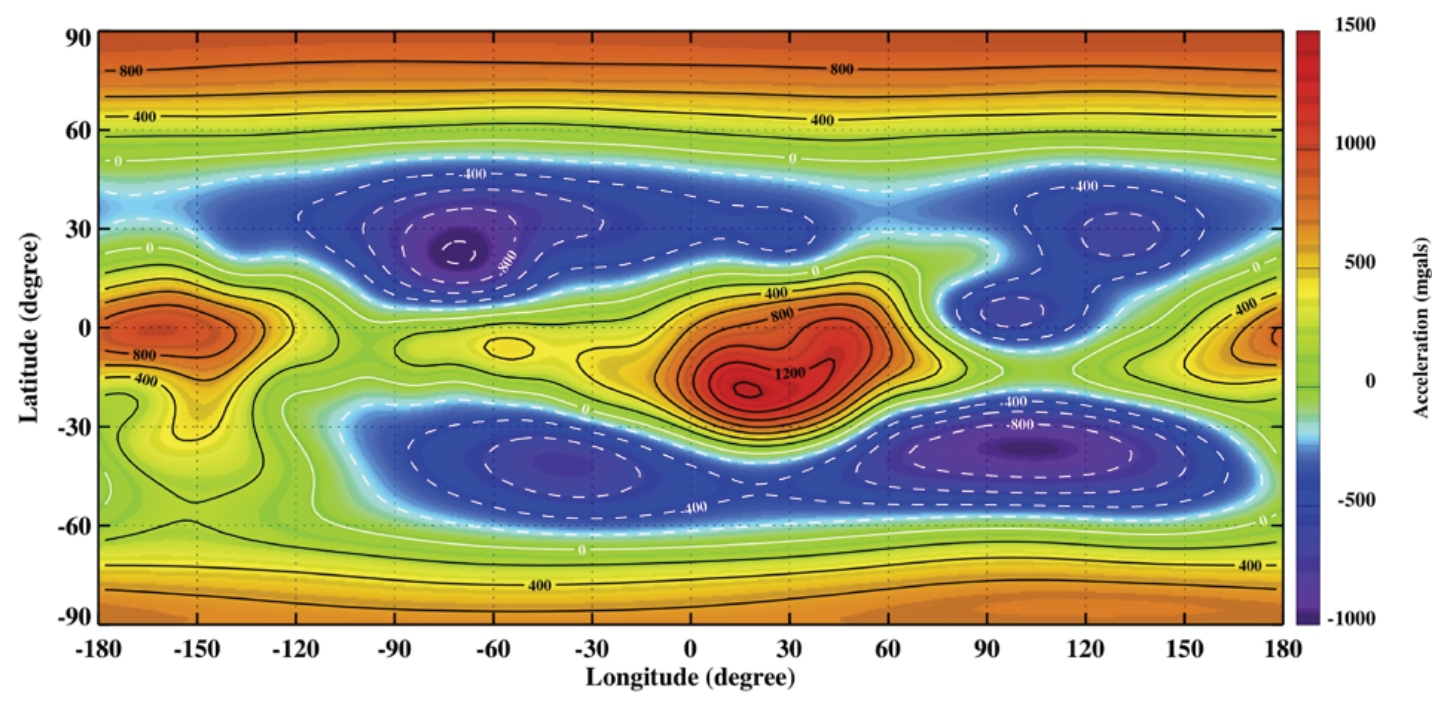

Fig. 8 The accelerations on a 290-km sphere for the 3-layered Vesta gravity model, a 15th degree spherical harmonic gravity model based upon the shape of Thomas et al. (1997). The acceleration due to $J_{2}$ is removed and the maximum and minimum accelerations are 1424 and -1024 mGals, respectively 


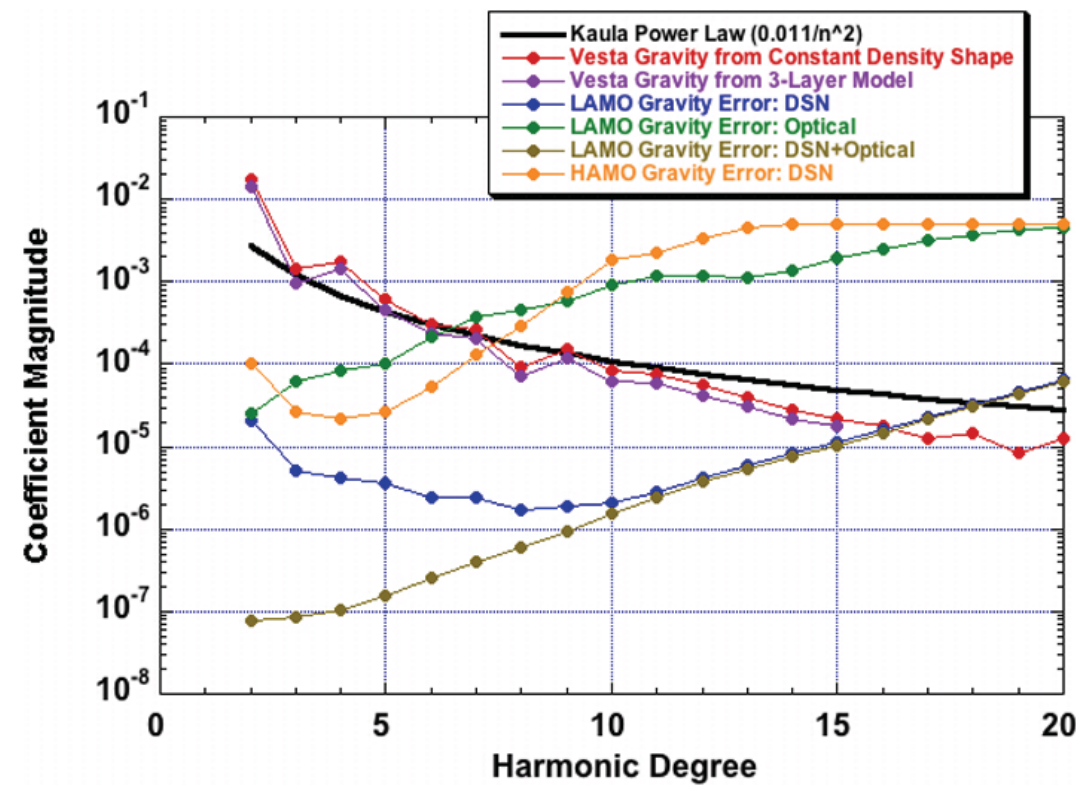

Fig. 9 The gravity RMS magnitude spectrum for Vesta simulations of one-month duration. The following spectrums are shown: the expected Kaula power law, gravity from shape assuming a constant density, gravity from shape with a 3-layer model, expected errors from the HAMO science phase using DSN tracking only, expected errors from the LAMO science phase using DSN data only, optical landmark tracking only, and DSN data together with optical landmark tracking. The amount of tracking included in the DSN data is $5.5 \mathrm{~h} /$ day

The expected power in the spherical harmonic coefficients are given by scaling the Earth's power law by (Kaula 1963)

$$
K_{\text {Vesta }}=K_{\text {Earth }}\left(\frac{M_{\text {Earth }}}{M_{\text {Vesta }}}\right)^{2}\left(\frac{R_{\text {Vesta }}}{R_{\text {Earth }}}\right)^{4}
$$

which gives $K_{\text {Vesta }}=0.011$ for $G M_{V e s t a}=17.66 \mathrm{~km}^{3} / \mathrm{s}^{2}$ (Konopliv et al. 2011) and $R_{\text {Vesta }}=265 \mathrm{~km}$ (Thomas et al. 1997). For Vesta, the gravity from shape contributions at low degree $(n<\sim 10)$ are greater than the expected power law, but drops off at higher degree probably due to loss of image resolution (Fig. 9). The gravity spectrums from various layered and shifted core models are similar in size (Tricarico and Sykes 2010).

After the Vesta Survey orbit at a radius of $2700-\mathrm{km}$, Dawn will be placed in HAMO1 for 29 days for the first acquisition of gravity data during September 2011. The orbit is polar and circular with a radius of $950-\mathrm{km}$ and an orbit period of about $12 \mathrm{~h}$. At this altitude, the $G M$ of Vesta is determined to $\pm 0.02 \mathrm{~km}^{3} / \mathrm{s}^{2}$ (10× formal uncertainties), and the low degree harmonics of the gravity field are significantly determined through harmonic degree 6 (Fig. 9). These HAMO results are from DSN data only with assumed $5.5 \mathrm{~h} /$ day of X-band $0.1 \mathrm{~mm} / \mathrm{s} 60$-s samples.

Next, after an orbit transition period, the Dawn spacecraft begins LAMO at Vesta on December 12, 2011 in a polar circular orbit. With an orbit semi-major axis of 460-km and orbit period of 4 hours, the average altitude of the Dawn spacecraft over the Vesta surface (mean $265-\mathrm{km}$ radius, Thomas et al. 1997) is about $200 \mathrm{~km}$. Accounting for the shape of Vesta, the average altitude over the north and south pole is about $200-\mathrm{km}$ and $250-\mathrm{km}$, respectively. The altitude difference is due to the shape of Vesta $(25-\mathrm{km}$ and $45-\mathrm{km}$ below the reference radius for the north and south pole, respectively) and orbit eccentricity (30 km higher in the south). For a given latitude, the altitude of the spacecraft can vary within a $60-\mathrm{km}$ 
range due to the strong irregular gravity field of Vesta, which is currently derived from the 3-layer Vesta gravity model. Tricarico and Sykes (2010) have shown the altitude variations are similar for other gravity models including constant density from shape (Thomas et al. 1997).

The expected quality of the LAMO gravity field determination at Vesta was investigated using 28-days of DSN Doppler tracking and optical landmark images prior to March 1, 2012. The LAMO radiometric (DSN) only, optical only and radiometric plus optical landmark tracking gravity error spectrums are displayed in Fig. 9. The gravity performance results assume $5.5 \mathrm{~h} /$ day X-band tracking with $0.1 \mathrm{~mm} / \mathrm{s}$ accuracy at 60 -s samples (easily achieved with planned HGA and LGA with DSN 70-m antennas), and the optical data assumes 20 images over 20 minutes once per day with 1.0 to 0.4 pixel resolution for an average of 30 tracking landmarks per picture. With both DSN and optical data and orbit variability assumptions, the spherical harmonic gravity field can be determined to about degree and order 20 (or 41-km half wavelength resolution at the surface of Vesta). These results provide ample margin for the $90-\mathrm{km}$ or spherical harmonic degree 9 gravity requirement. In addition, the $G M$ determination is at least $10 \times$ better than the HAMO expected results. Uncertainties for periodic signatures in $C_{21}$ and $S_{21}\left(10^{-7}\right.$, and consistent with the DSN plus optical solution of Fig. 9) indicate that wobble detection is possible for signatures on the order of $10-\mathrm{cm}$. The gravity information is primarily from the DSN Doppler data. The optical data, however, noticeably helps the long wavelength gravity field and orbit determination, as was the case for the NEAR mission (Konopliv et al. 2002).

The Dawn mission at Vesta will also acquire continuous DSN tracking during LAMO using the LGA on the spacecraft to the 34-m DSN stations. Figure 10 demonstrates the improvement for this scenario with 28 days of tracking assuming the data quality from the low gain is comparable to the high gain antenna. The expected resolution improves to harmonic degree 27 (or 30-km on the surface). A surprising RMS uncertainty curve results from this case, and is probably tied to large variations in the orbit. Plots of gravity coefficient uncertainty versus order reveals better determined higher order terms (near sectoral) for coefficients of low degree (e.g., $n=5$ ). For higher degree terms, the low order (near zonal) coefficients are better determined. Expected results should be between the two cases of 5.5-h/day and continuous tracking.

The orientation of the Vesta pole and its rotation rate are also determined as part of the solution for the gravity field through the dynamics of the spacecraft orbit. This solution is independent from the imaging team determination (Raymond et al. 2011b). The uncertainties in right ascension and declination of the pole (Seidelmann et al. 2007) are determined to better than $0.01^{\circ}$ with DSN data only, whereas inclusion of the landmark tracking improves pole location by at least $5 \times$ (see Table 3 ). In contrast, the landmark tracking significantly improves $(1000 \times)$ the rotation rate solution versus DSN only solutions and improves $(10 \times)$ the current knowledge (Drummond et al. 1988). As for previous gravity studies (e.g., Konopliv et al. 2006), the quoted uncertainties are $10 \times$ formal uncertainties to give realistic uncertainties that account for the red noise characteristics (power spectrum $f^{-8 / 3}$ ) of the Doppler data (Woo 1975) and other possible systematic errors such as modeled non-gravitational accelerations on the spacecraft. Any possible free wobble can be detected through time varying changes in the $C_{21}$ and $S_{21}$ gravity coefficients. The size of a detectable wobble for Vesta with period of 1.2 days (see above) is on the order of $20-\mathrm{cm}$ on the surface ( $r=265 \mathrm{~km}$; see Table 3). 

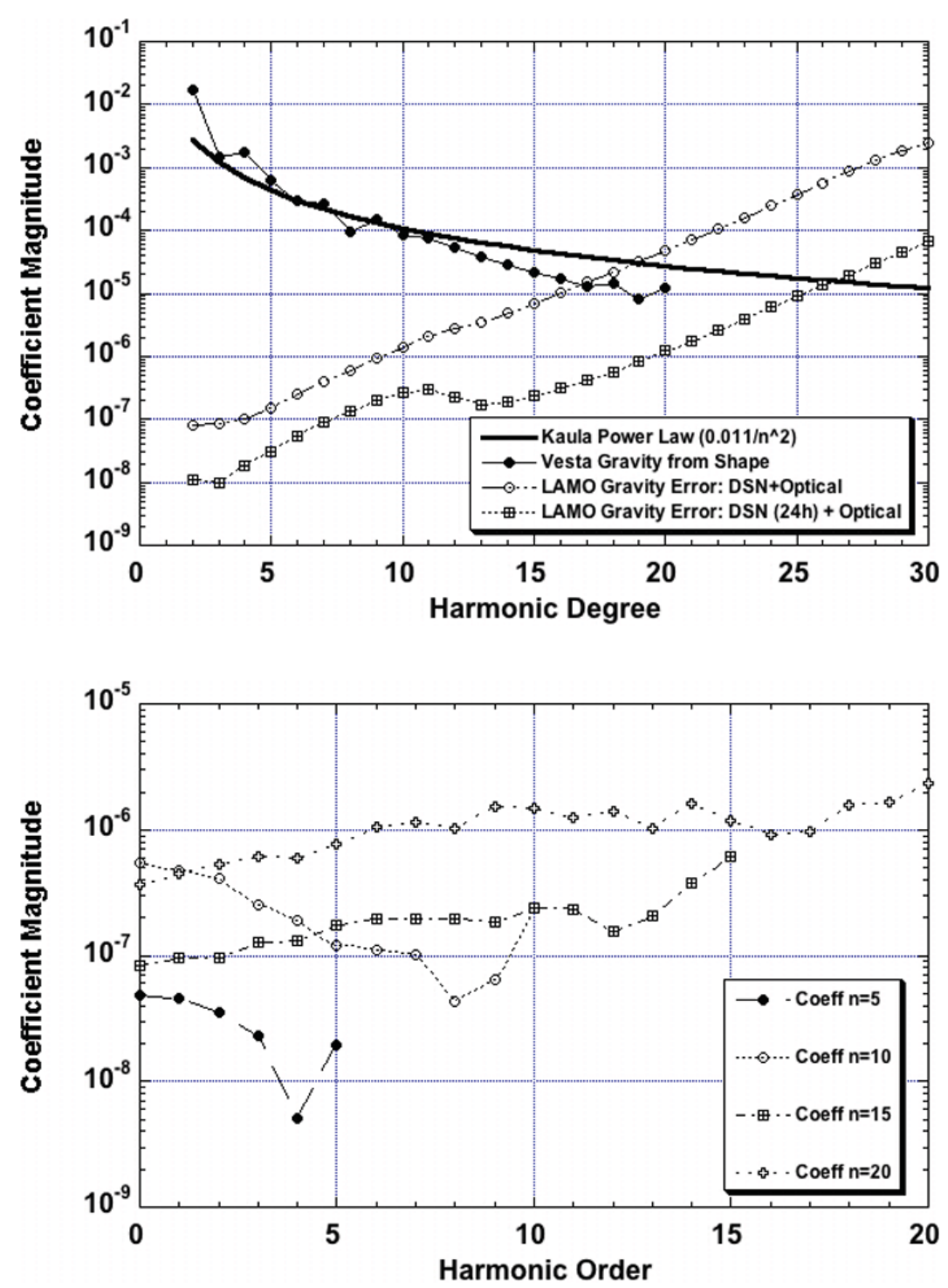

Fig. 10 The Vesta gravity error spectrums for the case with continuous (24 h) DSN tracking versus (a) harmonic degree and (b) harmonic order. Continuous tracking is an option for Dawn using the low gain antenna. This option improves the resolution from harmonic degree 19 to degree 27, and reduces the uncertainty of the determined coefficients by about an order of magnitude. The errors versus harmonic order are shown individually for degrees $5,10,15$, and 20

\section{Expected Gravity Science Results at Ceres}

The gravity field at Ceres is expected to be much smoother than Vesta because of small excursions $(<10-20 \mathrm{~km})$ from its nearly hydrostatic shape (Thomas et al. 1997; Carry et al. 2008) and lower density. In addition to the $G M$, the only a priori estimates for the Ceres gravity field come from the scaling of the Kaula power law $\left(K_{\text {Ceres }}=0.0086\right.$, using $R_{\text {Ceres }}=$ $476 \mathrm{~km}, G M_{\text {Ceres }}=63.2 \mathrm{~km}^{3} / \mathrm{s}^{2}$ ) and predictions of the $J_{2}$ value coming from the measured flattening and assumptions of hydrostatic equilibrium and internal structure. The values in unnormalized $J_{2}$ range from 0.016 for more differentiated models with a core to 0.032 for a homogeneous body (McCord and Sotin 2005; Zolotov 2009).

The Ceres survey orbit with a radius of $6400 \mathrm{~km}$ begins February 18, 2015 and lasts for 8 days (1.75 orbits). After a transfer phase, the HAMO mission phase begins May 2, 2015 
Table 3 Vesta and Ceres expected uncertainties in orientation from the Dawn mission. The uncertainties are for only the LAMO mission phase and the formal uncertainties are scaled by $10 \times$ to give more realistic values. The current values used as given as well as a recent pole solution for Vesta

\begin{tabular}{|c|c|c|c|c|c|}
\hline Asteroid & $\begin{array}{l}\text { R. A., } \alpha \\
\text { (deg) }\end{array}$ & $\begin{array}{l}\text { Dec., } \delta \\
\text { (deg) }\end{array}$ & $\begin{array}{l}\text { Rot. rate, } \omega \\
\text { (deg/day) }\end{array}$ & $\begin{array}{l}\text { Wobble } \\
\text { amp. }(\mathrm{cm})\end{array}$ & Notes \\
\hline \multirow[t]{5}{*}{ Vesta } & \pm 0.0040 & \pm 0.0057 & \pm 0.00043 & \pm 330 & DSN 5.5 h/day \\
\hline & \pm 0.0007 & \pm 0.0008 & \pm 0.0000003 & \pm 300 & $\begin{array}{l}\text { DSN } 5.5 \mathrm{~h} / \text { day }+ \\
\text { Optical Landmarks }\end{array}$ \\
\hline & \pm 0.0002 & \pm 0.0003 & \pm 0.0000001 & \pm 24 & $\begin{array}{l}\text { DSN } 24 \text { h/day }+ \\
\text { Optical Landmarks }\end{array}$ \\
\hline & $301 \pm 5$ & $41 \pm 5$ & $\begin{array}{l}1617.332776 \pm \\
0.000001\end{array}$ & & $\begin{array}{l}\text { Current values } \\
\text { Thomas et al. (1997) } \\
\text { Drummond et al. (1988) }\end{array}$ \\
\hline & $305.8 \pm 3.1$ & $41.4 \pm 1.5$ & & & $\begin{array}{l}\text { Recent Values } \\
\text { Li et al. (2011) }\end{array}$ \\
\hline \multirow[t]{2}{*}{ Ceres } & \pm 0.098 & \pm 0.0071 & \pm 0.00075 & & DSN 5.5 h/day \\
\hline & $291 \pm 5$ & $59 \pm 5$ & $\begin{array}{l}952.15320 \pm \\
0.00021\end{array}$ & & $\begin{array}{l}\text { Current values } \\
\text { Thomas et al. (2005) } \\
\text { Chamberlain et al. (2007) }\end{array}$ \\
\hline
\end{tabular}

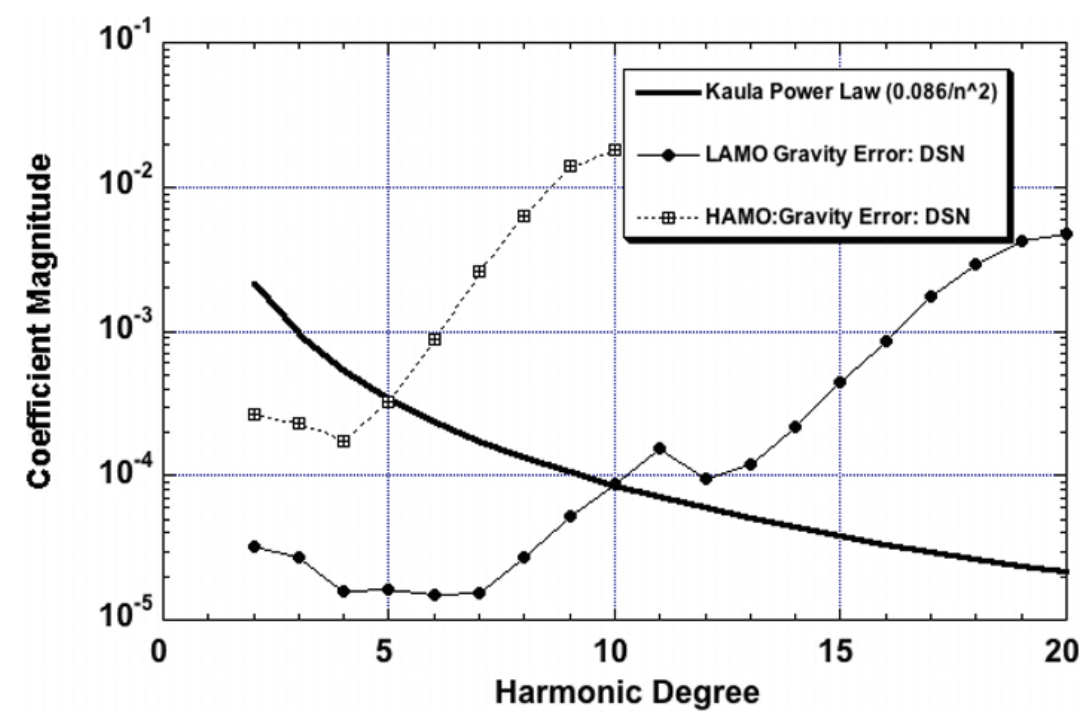

Fig. 11 The gravity RMS magnitude spectrum for the Ceres one-month simulation. The following spectra are shown: the expected Kaula power law, the HAMO science phase expected results, and the LAMO science phase expected results. These results are for DSN tracking only at $5.5 \mathrm{~h} /$ day. Optical landmark tracking is not part of the simulation at this time, but it is expected to improve results in similar fashion as with Vesta

and lasts for 21 days in an orbit with radius $1800-\mathrm{km}$ and a period of 16.8 hours. Since the HAMO mission phase occurs with a small Sun angle (Doppler noise $\sim 5 \times$ worse), the $G M$ of Ceres is not well determined with an uncertainty of about $\pm 0.2 \mathrm{~km}^{3} / \mathrm{s}^{2}$. For the same reason, the spherical harmonics solution for the HAMO phase is degraded as shown in Fig. 11, where the coefficients are determined through degree 4 and $J_{2}$ (unnormalized) is determined to about $3 \%(10 \times$ formal uncertainty). These results are for Doppler tracking only with $5.5 \mathrm{~h}$ of X-band data per day at $0.5 \mathrm{~mm} / \mathrm{s}$ accuracy (degraded for the Sun angle). The $G M$ and pole determination of the orbit depends on the nonspherical nature of the 
gravity field. For our simulation a nominal $0.03 J_{2}$ value (unnormalized) is used with the Earth gravity field scaled to Ceres used for the remaining coefficients.

The Ceres LAMO mission phase begins June 22, 2015 and lasts for 30 days in a polar circular orbit. The orbit radius is $1180 \mathrm{~km}$ resulting in an orbit period of 8.9 hours. The Sun angle is more favorable for this part of the mission and so a better accuracy of $0.1 \mathrm{~mm} / \mathrm{s}$ for the Doppler data can be used. With this orbit and tracking accuracy, the gravity field can be determined to degree and order 10, or a surface resolution of $140 \mathrm{~km}(<300 \mathrm{~km}$ requirement). As in HAMO, these results assume DSN tracking only with 5.5 hours per day. The optical imaging landmark tracking scenario has yet to be simulated for Ceres. However, similar improvement as with Vesta is expected in the long-wavelength gravity uncertainties. The uncertainty in the $G M$ of Ceres reduces in the LAMO orbit to $\pm 0.02 \mathrm{~km}^{3} / \mathrm{s}^{2}(10 \times$ formal), and the $J_{2}$ uncertainty reduces to $0.4 \%$. Both these results should improve $(\sim 10 \times)$ with optical landmark tracking and if additional Doppler tracking is available.

The Ceres pole and rotation rate uncertainties expected from the HAMO mission phase are given in Table 3. Results are only presented for DSN tracking only, and uncertainties are expected to improve similar to Vesta $(\sim 5 \times$ for pole and up to $1000 \times$ for the spin rate) once landmark tracking observables are incorporated into the analysis. Additional DSN tracking via the LGA represents another possible improvement for Ceres.

Acknowledgements Bob Werner provided polyhedral software and assistance for the gravitational modeling of multilayered Vesta shape models. The research described in this paper was carried out at the Jet Propulsion Laboratory, California Institute of Technology, under contract with the National Aeronautics and Space Administration.

\section{References}

J.D. Anderson, E.L. Lau, W.L. Sjogren, G. Schubert, W.B. Moore, Gravitational constraints on the internal structure of Ganymede. Nature 384, 541-543 (1996)

J.D. Anderson, E.L. Lau, W.L. Sjogren, G. Schubert, W.B. Moore, Europa's differentiated internal structure: inferences from four Galileo encounters. Science 281, 2019-2022 (1998a)

J.D. Anderson, G. Schubert, R.A. Jacobson, E.L. Lau, W.B. Moore, W.L. Sjogren, Distribution of rock, metals, and ices in Callisto. Science 280, 1573-1576 (1998b)

J.D. Anderson, R.A. Jacobson, E.L. Lau, W.B. Moore, G. Schubert, Io's gravity field and interior structure. J. Geophys. Res. 106, 32963-32969 (2001)

S.W. Asmar, J.W. Armstrong, L. Iess, P. Tortora, Spacecraft Doppler tracking: noise budget and achievable accuracy in precision radio science observations. Radio Sci. 40, RS2001 (2005). doi:10.1029/ 2004RS003101

J. Baer, A. Milani, S.R. Chesley, R.D. Matson, An observational error model, and application to asteroid mass determination. Bull. Am. Astron. Soc. 40, 493 (2008)

G.J. Bierman, Factorization Methods for Discrete Sequential Estimation (Academic Press, New York, 1977)

G.G. Bills, Discrepant estimates of moments of inertia of the Moon. J. Geophys. Res. 100, 26,297-26,303 (1995)

B.G. Bills, D.P. Rubincam, Constraints on density models from radial moments: applications to Earth, Moon, and Mars. J. Geophys. Res. 100, 26305-26315 (1995)

B.G. Bills, F. Nimmo, Forced obliquity and moments of inertia of Titan. Icarus 196, 293-297 (2008)

B.G. Bills, F. Nimmo, Forced obliquities and moments of inertia of Ceres and Vesta. Icarus 213, 496-509 (2011)

G.J. Black, P.D. Nicholson, W.F. Bottke, J.A. Burns, A.W. Harris, On a possible rotation state of 433 Eros. Icarus 140, 239-242 (1999)

W.F. Bottke, D. Vokrouhlicky, D.P. Rubincam, D. Nesvorny, The Yarkovsky and YORP effects: implications for asteroid dynamics. Annu. Rev. Earth Planet. Sci. 34, 157-191 (2006)

J.A. Burns, V.S. Safronov, Asteroid nutation angles. Mon. Not. R. Astron. Soc. 165, 403-411 (1973)

B. Carry, C. Dumas, M. Fulchignoni, W.J. Merline, J. Berthier, D. Hestroffer, T. Fusco, P. Tamblyn, Nearinfrared mapping and physical properties of the Dwarf-planet Ceres. Astron. Astrophys. 478, 235-244 (2008) 
M.A. Chamberlain, M.V. Sykes, G.A. Esquerdo, Ceres lightcurve analysis—period determination. Icarus 188, 451-456 (2007)

W. Chen, W. Shen, J. Han, J. Li, Free wobble of the triaxial Earth: theory and comparisons with International Earth rotation service data. Surv. Geophys. 30, 39-49 (2009)

G. Colombo, Cassini's second and third laws. Astron. J. 71, 891-900 (1966)

G.H. Darwin, Theory of the figure of the Earth carried to the second order of small quantities. Mon. Not. R. Astron. Soc. 60, 82-124 (1899)

J. Drummond, A. Eckhart, E.K. Hege, Speckle interferometry of asteroids. IV. Reconstructed images of 4 Vesta. Icarus 73, 1-14 (1988)

A. Fienga, J. Laskar, T. Morley, H. Manche, P. Kuchynka, C. Le Poncin-Lafitte, F. Budnik, M. Gastineau, L. Somenzi, INPOP08: a 4-D planetary ephemeris: from asteroid and time-scale computations to ESA Mars Express and Venus Express contributions. Astron. Astrophys. 507, 1675-1686 (2009)

W.M. Folkner, P. Charlot, M.H. Finger, J.G. Williams, O.J. Sovers, X.X. Newhall, E.M. Standish, Determination of the extragalactic-planetary frame tie from joint analysis of radio interferometric and lunar laser ranging measurements. Astron. Astrophys. 287, 279-289 (1994)

W.M. Folkner, R.D. Kahn, R.A. Preston, C.F. Yoder, E.M. Standish, J.G. Williams, C.D. Edwards, R.W. Hellings, T.M. Eubanks, B.G. Bills, Mars dynamics from Earth-based tracking of the Mars Pathfinder lander. J. Geophys. Res. 102, 4057-4064 (1997)

R. Garmier, J.P. Barriot, Ellipsoidal harmonic expansions of the gravitational potential: theory and Application. Celest. Mech. Dyn. Astron. 79, 235-275 (2001)

R. Garmier, J.P. Barriot, A.S. Konopliv, D.K. Yeomans, Modeling of the Eros gravity field as an ellipsoidal harmonic expansion from the NEAR Doppler tracking data. Geophys. Res. Lett. 29 (2002). doi:10.1029/2001GL013768

R.W. Gaskell, Landmark navigation and target characterization in a simulated Itokawa encounter, in AAS/AIAA Astrodynamics Specialists Conf., Lake Tahoe, CA (2005). AAS paper 05-289

R.W. Gaskell, O.S. Barnouin-Jha, D.J. Scheeres, A.S. Konopliv, T. Mukai, S. Abe, J. Saito, M. Ishiguro, T. Kubota, T. Hashimoto, J. Kawaguchi, M. Yoshikawa, K. Shirakawa, T. Kominato, N. Hirata, H. Demura, Characterizing and navigating small bodies with imaging data. Meteorit. Planet. Sci. 43, 1049-1061 (2008)

K. Gwinner, F. Scholten, F. Preusker, S. Elgner, T. Roatsch, M. Spiegel, R. Schmidt, J. Oberst, R. Jaumann, C. Heipke, Topography of Mars from global mapping by HRSC high-resolution digital terrain models and orthoimages: characteristics and performance. Earth Planet. Sci. Lett. 294, 506-519 (2010)

A.W. Harris, Tumbling asteroids. Icarus 107, 209-211 (1994)

W.A. Heiskanen, H. Moritz, Physical Geodesy (Freeman, San Francisco, 1967)

J. Henrard, C. Murigande, Colombo's top. Celest. Mech. Dyn. Astron. 40, 345-366 (1987)

W.B. Hubbard, J.D. Anderson, Possible flyby measurements of Galilean satellite interior structure. Icarus 33, 336-341 (1978)

W.M. Kaula, The investigation of the gravitational fields of the Moon and planets with artificial satellites. Adv. Space Sci. Technol. 5, 210-230 (1963)

W.M. Kaula, Theory of Satellite Geodesy (Blaisdell, Waltham, 1966)

K. Keil, Geological history of asteroid 4 Vesta: the "smallest terrestrial planet", in Asteroids III, ed. by W. Bottke, A. Cellino, P. Paolicchoi, R.P. Binzel (University of Arizona Press, Tucson, 2002), pp. 573-584

A.S. Konopliv, J.K. Miller, W.M. Owen, D.K. Yeomans, J.D. Giorgini, R. Garmier, J.P. Barriot, A global solution for the gravity field, rotation, landmarks, and ephemeris of Eros. Icarus 160, 289-299 (2002)

A.S. Konopliv, C.F. Yoder, E.M. Standish, D.N. Yuan, W.L. Sjogren, A global solution for the Mars static and seasonal gravity, Mars orientation, Phobos and Deimos masses, and Mars ephemeris. Icarus 182, 23-50 (2006)

A.S. Konopliv, S.W. Asmar, W.M. Folkner, O. Karatekin, D.C. Nunes, S.E. Smrekar, C.F. Yoder, M.T. Zuber, Mars high resolution gravity fields from MRO, Mars seasonal gravity, and other dynamical parameters. Icarus 211, 401-428 (2011)

F.T. Krogh, Changing stepsize in the integration of differential equations using modified divided differences. JPL Technical Memorandum No. 312, Section 914 (internal document), Jet Propulsion Laboratory, California Institute of Technology, Pasadena, CA (1973)

M. Kuzmanoski, G. Apostolovska, B. Novaković, The mass of (4) Vesta derived from its largest gravitational effects. Astron. J. 140(3), 880-886 (2010). doi:10.1088/0004-6256/140/3/880

K. Lambeck, Earth's Variable Rotation: Geophysical Causes and Consequences (Cambridge Univ. Press, Cambridge, 1980)

K. Lambeck, Geophysical Geodesy (Clarendon, Oxford, 1988)

C.L. Lawson, R.J. Hanson, Solving Least Squares Problems. SIAM Classics in Applied Mathematics, vol. 15 (Society for Industrial and Applied Mathematics, Philadelphia, 1995) 
J.-Y. Li, P.C. Thomas, B. Carcich, M.J. Mutchler, L.A. McFadden, C.T. Russell, S.S. Weinstein-Weiss, M.D. Rayman, C.A. Raymond, Improved measurement of asteroid (4) Vesta's rotational axis orientation. Icarus 211, 528-534 (2011)

N. Mastrodemos, B. Rush, D. Vaughan, W. Owen, Optical navigation for Dawn at Vesta, in 21st AAS/AIAA Space Flight Mechanics Meeting, New Orleans, LA (2011). AAS Paper 11-222

T.B. McCord, C. Sotin, Ceres: Evolution and current state. J. Geophys. Res. 110, E05009 (2005). doi:10.1029/2004JE002244

T.B. McCord et al., Origin, evolution and structure of Ceres. Space Sci. Rev. (2011, this issue). doi:10.1007/s11214-010-9729-9

G. Michalak, Determination of asteroid masses. I. (1) Ceres, (2) Pallas, and (4) Vesta. Astron. Astrophys. 360, 363-374 (2000)

T.D. Moyer, Formulation for observed and computed values of deep space network data types for navigation. Monograph 2. Deep Space Communications and Navigation Series, JPL Publication 00-7, Jet Propulsion Laboratory, California Institute of Technology, Pasadena, CA (2000)

T.D. Moyer, Mathematical formulation of the double-precision orbit determination program (DPODP). JPL technical report 32-1527, Jet Propulsion Laboratory, California Institute of Technology, Pasadena, CA (1971)

W.H. Munk, G.J.F. MacDonald, The Rotation of the Earth (Cambridge Univ. Press, New York, 1960)

S.M. Nakiboglu, Hydrostatic theory of the Earth and its mechanical implications. Phys. Earth Planet. Inter. 28, 302-311 (1982)

G.A. Neumann, M.T. Zuber, D.E. Smith, F.G. Lemoine, The lunar crust: global signature and structure of major basins. J. Geophys. Res. 101, 16,841-16,863 (1996)

S.J. Peale, Excitation and relaxation of the wobble, precession, and libration of the Moon. J. Geophys. Res. 81, 1813-1827 (1976)

S.J. Peale, Generalized Cassini's laws. Astron. J. 74, 483-490 (1969)

S.J. Peale, The free precession and libration of Mercury. Icarus 178, 4-18 (2005)

E.V. Pitjeva, High-precision ephemerides of planets-EPM and determination of some astronomical constants. Sol. Syst. Res. 39, 176-186 (2005)

E.V. Pitjeva, E.M. Standish, Proposals for the masses of the three largest asteroids, the Moon-Earth mass ratio and the Astronomical Unit. Celest. Mech. Dyn. Astron. 103, 365-372 (2009)

C. Polansky et al., Science operations and planning, Space Sci. Rev. (2011, this issue)

R. Radau, Sur la loi des densites a l'interieur de la Terre. Compt. Rend. 100, 972-974 (1885)

M.D. Rayman et al., Mission design, Space Sci. Rev. (2011a, this issue)

C.A. Raymond et al., Dawn topography investigation, Space Sci. Rev. (2011b, this issue)

M.G. Rochester, D.E. Smylie, On changes in the trace of Earth's inertia tensor. J. Geophys. Res. 79, 49484951 (1974)

N.H. Samarasinha, Rotational excitation and damping as probes of interior structures of asteroids and comets. Meteorit. Planet. Sci. 43, 1063-1073 (2008)

D.J. Scheeres, S. Mirrahimi, Rotational dynamics of a solar system body under solar radiation torques. Celest. Mech. Dyn. Astron. 101, 69-103 (2008)

P.K. Seidelmann, B.A. Archinal, M.F. A’hearn, A. Conrad, G.J. Consolmagno, D. Hestroffer, J.L. Hilton, G.A. Krasinsky, G. Neumann, J. Oberst, P. Stooke, E.F. Tedesco, D.J. Tholen, P.C. Thomas, I.P. Williams, Report of the IAU/IAG Working Group on cartographic coordinates and rotational elements: 2006. Celest. Mech. Dyn. Astron. 98, 155-180 (2007)

I. Sharma, J.A. Burns, C.Y. Hui, Nutational damping times in solids of revolution. Mon. Not. R. Astron. Soc. Lett. 359, 79-92 (2005)

H. Sierks et al., The Dawn framing camera. Space Sci. Rev. (2011, this issue). doi:10.1007/11214011-9745-4

S.M. Slivan, Spin vector alignment of Koronis family asteroids. Nature 419, 49-51 (2002)

S.M. Slivan, R.P. Binzel, L.D. Crespo da Silva, M. Kaasalainen, M.M. Lyndaker, M. Krco, Spin vectors in the Koronis family: comprehensive results from two independent analyses of 213 rotation lightcurves. Icarus 162, 285-307 (2003)

S.M. Slivan, R.P. Binzel, M. Kaasalainen, A.N. Hock, A.J. Klesman, L.J. Eckelman, R.D. Stephens, Spin vectors in the Koronis family. II. Additional clustered spins, and one stray. Icarus 200, 514-530 (2008)

M.L. Smith, F.A. Dahlen, Period and Q of the Chandler wobble. Geophys. J. R. Astron. Soc. 64, 223-281 (1981)

R. Sniffin, S. Slobin, D. Morabito, A. Bedrossian, D. Shin, L. Paul, DSMS Telecommunications Link Design Handbook, Rev. E (Jet Propulsion Laboratory, Pasadena, 2000)

T. Soler, A new matrix development of the potential at exterior points as a function of the inertia tensor. Celest. Mech. 32, 257-296 (1984) 
E.M. Standish, Suggested GM values for Ceres, Pallas, and Vesta. JPL interoffice memorandum (internal document), 312.F-01-006, April 11, 2001

B.D. Tapley, B.E. Schutz, G.H. Born, Statistical Orbit Determination (Elsevier, Boston, 2004)

F.H. Taylor, DESCANSO design and performance summary series. In: Dawn Telecommunications, Article 13, Jet Propulsion Laboratory, Pasadena, California, August 2009

P.C. Thomas, R.P. Binzel, M.J. Gaffey, B.H. Zellner, A.D. Storrs, E. Wells, Vesta: spin pole, size, and shape from HST Images. Icarus 128, 88-94 (1997)

P.C. Thomas, J.W. Parker, L.A. McFadden, C.T. Russell, S.A. Stern, M.V. Sykes, E.F. Young, Differentiation of the asteroid Ceres as revealed by its shape. Nature 437, 224-226 (2005)

P. Tricarico, M.V. Sykes, The dynamical environment of Dawn at Vesta. Planet. Space Sci. 58, 1516-1525 (2010)

D. Vokrouhlicky, D. Nesvorny, W.F. Bottke, The vector alignments of asteroid spins by thermal torques. Nature 425, 147-151 (2003)

D. Vokrouhlicky, D. Nesvorny, W.F. Bottke, Secular spin dynamics of inner main-belt asteroids. Icarus 184, 1-28 (2006)

B.P. Weiss, J.S. Berdahl, L. Elkins-Tanton, S. Stanley, E.A. Limea, L. Carporzen, Magnetism on the angrite parent body and the early differentiation of planetesimals. Science 322(5902), 713-716 (2008)

R.A. Werner, D.J. Scheeres, Exterior gravitation of a polyhedron derived and compared with harmonic and mascon gravitation representations of asteroid 4769 Castalia. Celest. Mech. Dyn. Astron. 65, 313-344 (1997)

M.A. Wieczorek, Gravity and topography of the terrestrial planets. Treat. Geophys. 10, 165-206 (2007). doi:10.1016/B978-044452748-6/00156-5

J.G. Williams, Contributions to the Earth's obliquity rate, precession, and nutation. Astron. J. 108, 711-724 (1994)

R. Woo, Multifrequency techniques for studying interplanetary scintillations. Astrophys. J. 201, 238-248 (1975)

M.Y. Zolotov, On the composition and differentiation of Ceres. Icarus 204, 183-193 (2009)

M.T. Zuber, D.E. Smith, F.G. Lemoine, G.A. Neumann, The shape and internal structure of the Moon from the Clementine mission. Science 266, 1839-1843 (1994)

M.T. Zuber, S.C. Solomon, R.J. Phillips, D.E. Smith, G.L. Tyler, O. Aharonson, G. Balmino, W.B. Banerdt, J.W. Head, F.G. Lemoine, P.J. McGovern, G.A. Neumann, D.D. Rowlands, S. Zhong, Internal structure and early thermal evolution of Mars from Mars Global Surveyor topography and gravity. Science 287, 1788-1793 (2000)

M.T. Zuber et al., Origin, evolution and structure of Vesta, Space Sci. Rev. (2011, this issue) 